\title{
El desarrollo regional en la República Popular China: de la administración centralizada a la planificación del desarrollo
}

\section{Focusing on Regional Development of the People's Republic of China: from centralized administration towards planning of development}

\author{
JianNan $\mathrm{Xu}^{*}$ y José Luis Yagüe Blanco**
}

\section{INTRODUCCIÓN}

El objetivo principal de este artículo es dar una visión general del proceso de toma en consideración de las regiones de la República Popular China (RPC). Desde la llegada de la nueva estructura política comunista la división administrativa del sistema chino se ha ajustado constantemente y aunque esto ha provocado profundos cambios sociales hasta nuestros días, aún se continúa buscando una reforma adecuada a los requerimientos del nuevo desarrollo. En este sentido, si queremos evaluar con precisión los éxitos y los fracasos es necesario el conocimiento riguroso de los orígenes de la división actual y de los intentos previos para regionalizar el país, principalmente desde el punto de vista de la división administrativa y de la política de desarrollo regional (Yagüe y Díaz-Puente, 2008).

A tal efecto, el análisis se divide en dos grandes períodos que ayudan a la comprensión de esta evolución. Pero previamente es preciso realizar una

\footnotetext{
* Máster en Planificación y Gestión Sostenible de Proyectos de Desarrollo Rural. Universidad Politécnica de Madrid.

${ }^{* *}$ Investigador contratado. Grupo de investigación en Planificación y Gestión Sostenible del Desarrollo Rural-Local (GESPLAN), Universidad Politécnica de Madrid (joseluis.yague@upm.es).
} 
breve introducción histórica hasta el momento en que se funda la Republica Popular China, lo cual nos permitirá conocer mejor la situación del país en la fase inicial del período de análisis considerado.

En los cinco mil años de historia de China, tomamos los periodos de Primavera y de Otoño (770-476 a. C.) como punto de partida de la estructuración regional, cuando China inició un sistema de administración local. En el año 221 a. C., el primer emperador de la dinastía Qin unió por primera vez todo el territorio de China e implementó el sistema de prefecturas y de condados por toda la nación, dividiendo el país en 36 prefecturas (Bu, 2009). Desde entonces, tanto el territorio como las divisiones administrativas de China han experimentado constantes cambios en las dinastías siguientes, hasta que la dinastía Qing (1636-1911) estableció la base del territorio de China que hoy conocemos (Hu, 2006; Gernet, 2006 y 2008) (figura 1).

El perfil topográfico de este territorio se conforma por tres pisos que descienden de oeste a este. Empieza en el suroeste, en la meseta de Qinghai-Tíbet, de 4.000 metros de altitud, tradicionalmente la zona más pobre. Luego, en la región central, la topografía desciende hasta las montañas y las cuencas

FIGURA 1

DIVISIÓN TERRITORIAL DE LA DINASTÍA QING (1840)

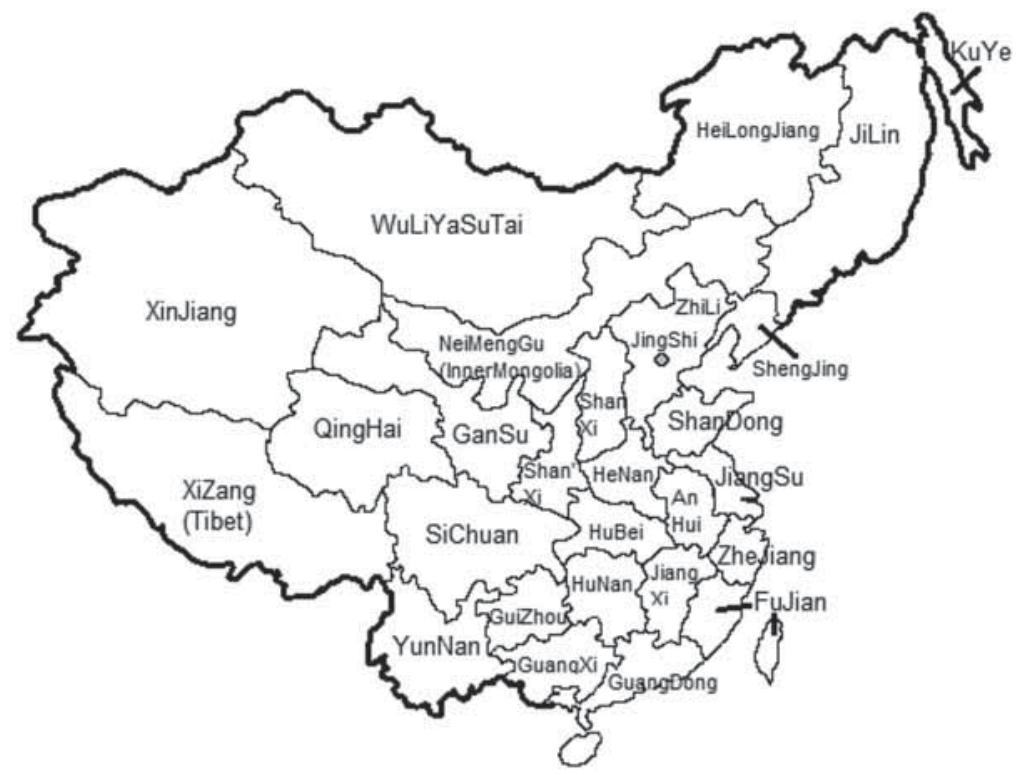

Fuente: elaboración propia a partir de China Map Press, 2009.

Estudios Geográficos, Vol. LXXIII, 272, pp. 273-307, enero-junio 2012

ISSN: 0014-1496, eISSN: 1988-8546, doi: 10.3989/estgeogr.201210 
que se despliegan desde los 2.000 a los 1.000 metros sobre el nivel del mar. Esa zona es rica en recursos minerales, pero son costosos de extraer y de transportar. Seguidamente, desciende hacia el este, hasta las regiones y las llanuras por debajo de los 1.000 metros. Estas regiones orientales tienen condiciones naturales más favorables para la agricultura y el comercio, lo que explica la mayor riqueza de esta región (Bao et al., 2002).

Desde la Guerra del Opio, declarada por Gran Bretaña en 1840, el gobierno Qing fue invadido constantemente por fuerzas extranjeras, firmando muchas cláusulas desiguales que ocasionaron la perdida de importantes territorios. En 1911 la Revolución Xinhai derrocó al Imperio y el año siguiente fue declarada la República de China (1912-1949). En los primeros años de la República el Gobierno Central fue débil e ineficaz, con docenas de «señores de la guerra» ocupando el vacío político, a menudo apoyados por alguna potencia extranjera (Gernet, 2006 y 2008; Roux, 2006) (figura 2).

\section{FIGURA 2}

DIVISIÓN TERRITORIAL DE LOS PRINCIPALES «SEÑORES DE LA GUERRA» EN LOS PRIMEROS AÑOS DE LA REPÚBLICA Y APOYO DE LAS POTENCIAS EXTRANJERAS

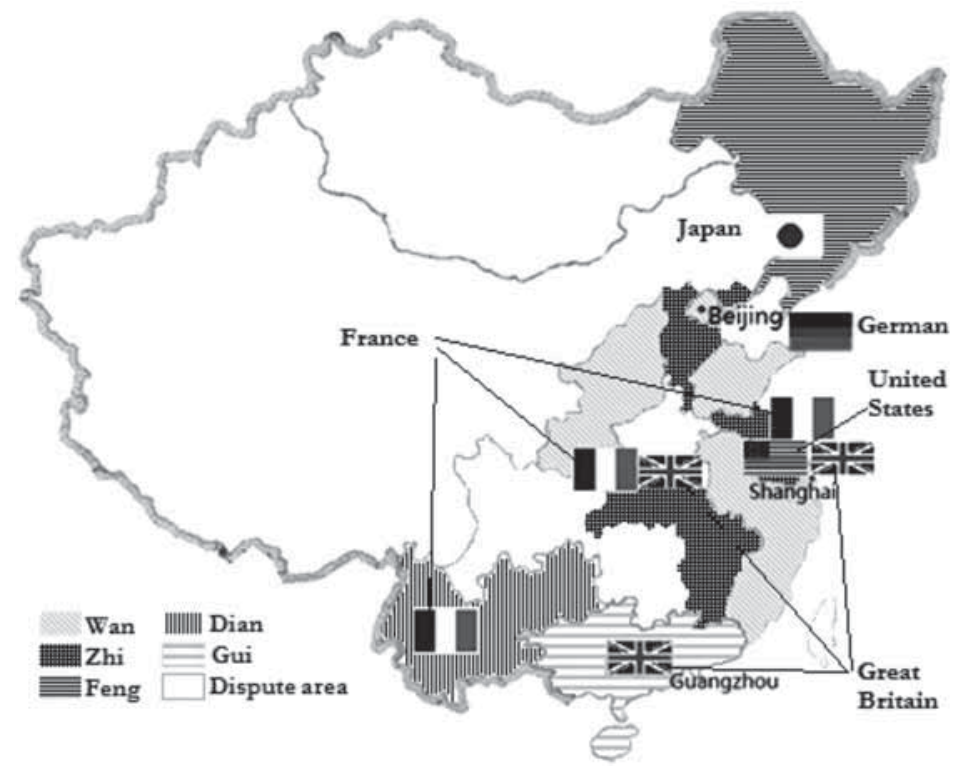

Fuente: elaboración propia a partir de History Department of People's Education Publishing House and Education Map Centre of China Map Press, 2003; Williams y Dietz, 1989. 
A lo largo la década de los años veinte, diferentes facciones militares chinas se encontraban en conflicto, mientras que Japón consolidaba su dominio al norte de la región de Manchuria (1932) y a lo largo de la costa de China (1937). Ya insertos plenamente en el conflicto mundial, el movimiento de resistencia protagonizado tanto por la facción nacionalista china como por la comunista, provocaron la rendición de Japón en agosto de 1945, momento que marca también el comienzo de la guerra civil en China. En noviembre de 1948, los comunistas liderados por Mao ZeDong tomaron el control del norte del país y en enero de 1949, el Partido Nacionalista dirigido por Jiang JieShi se retiró a Taiwán. El primero de octubre de 1949 se fundó la República Popular China (Williams y Dietz, 1989; Gernet, 2006 y 2008; Roux, 2006).

Bajo estas condiciones de guerra e incertidumbre, la sociedad experimentó dramáticas transformaciones cuyas consecuencias condicionaron la evolución

\section{FIGURA 3}

DIVISIÓN TERRITORIAL DE LA REPÚBLICA CHINA EN EL PERIODO 1912-1949

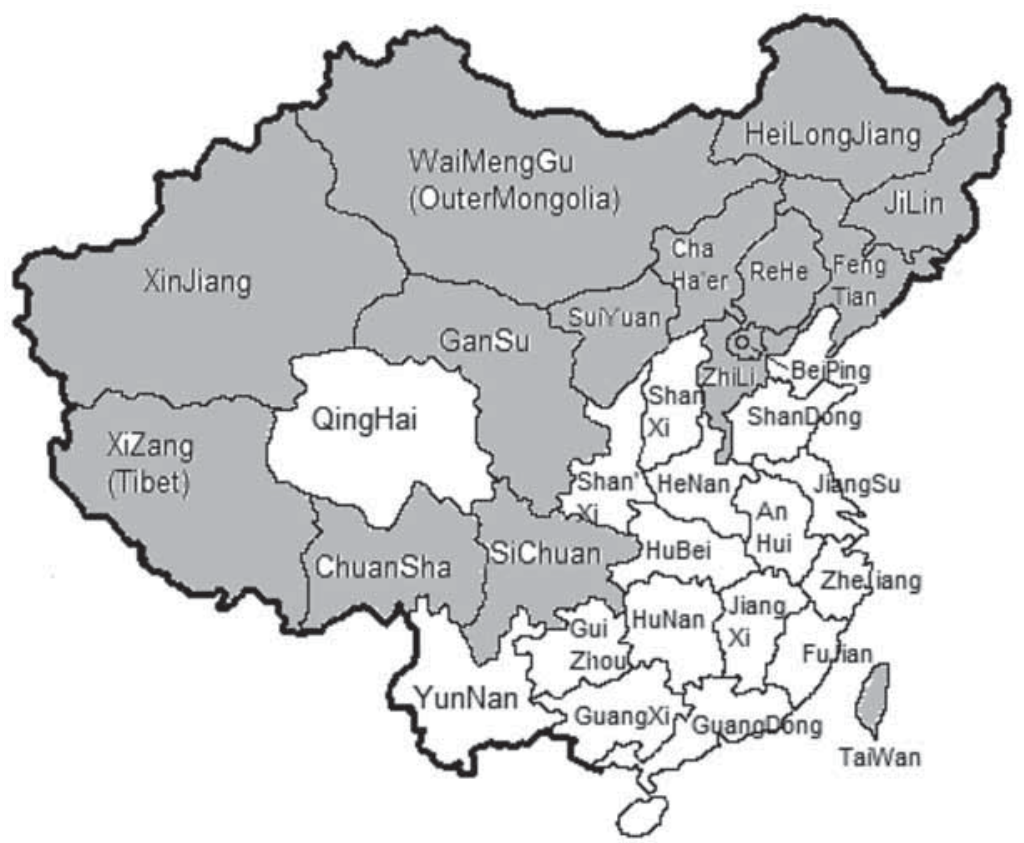

Nota: las provincias en color gris representan aquellas con cambios en sus límites en comparación con el periodo anterior.

Fuente: elaboración propia a partir de China Map Press, 2009. 
de la Republica Popular China en el futuro. A título de ejemplo — como se introdujo en la figura 2-, las fuerzas extranjeras siempre residieron en las zonas orientales y costeras por sus intereses comerciales. Sus negocios también influyeron en el desarrollo económico local e industrial. Esto contribuye en parte a explicar cómo la región costera del este de China presenta en la actualidad un mayor grado de desarrollo que la China media y occidental.

En cualquier caso, durante la mayor parte de su historia, la división administrativa de la República China se mantuvo como muestra la figura 3, que tomamos como punto de partida para el análisis. Hasta este momento más de cuatro millones de kilómetros cuadrados del norte y del nordeste de China fueron cedidos a Rusia; la provincia de Taiwán y otras islas a Japón, y el oeste del Tíbet a la India. Finalmente el norte y el nordeste del país fueron divididos en varias provincias en comparación con la dinastía Qing (Zhang, 1994).

\section{El Periodo del Mao}

El primer período contemplado abarca desde 1949 a 1978 y se caracteriza por reformas drásticas en la división administrativa y por la unificación del sistema financiero de ingresos y de gastos de acuerdo al nuevo sistema de administración centralizada (Chen y Zhang, 2009).

Aunque la República Popular China se fundó en 1949, la denominada Guerra de Liberación no terminó hasta 1952 (figura 4). En 1953, China formuló y ejecutó el Primer Plan Quinquenal inspirado en la planificación central de estilo soviético. De 1958 a 1961, el Partido Comunista lanzó la campaña del «Gran Salto Adelante» cuyo objetivo era desarrollar rápidamente la productividad social. En el mismo período, la producción en las zonas rurales fue transformada profundamente con la campaña denominada «Movimiento de Formación de Comunas de la Población Rural» que pretendía crear una comunidad igualitaria, media, justa y razonable sobre la base de una productividad que estaba infrautilizada. En los años de 1962-1966 Mao se retiró y China experimentó una recuperación y una reorientación de las campañas políticas, con un control más relajado. Pero en los siguientes diez años hasta 1976, Mao regresó y puso en marcha la «Revolución Cultural», que incluía una serie de campañas como la «Lucha Armada» y la «Destrucción de todos los Pensamientos Tradicionales, Culturas, Costumbres y Hábitos». Estas campañas dañaron seriamente el orden social y la vida normal hasta ese momento (Williams, 1994; Editorial Committee on Party Literature of the Central Committee, 1993). 
FIGURA 4

AVANCE DEL TERRITORIO LIBERADO DURANTE LA GUERRA DE LIBERACIÓN

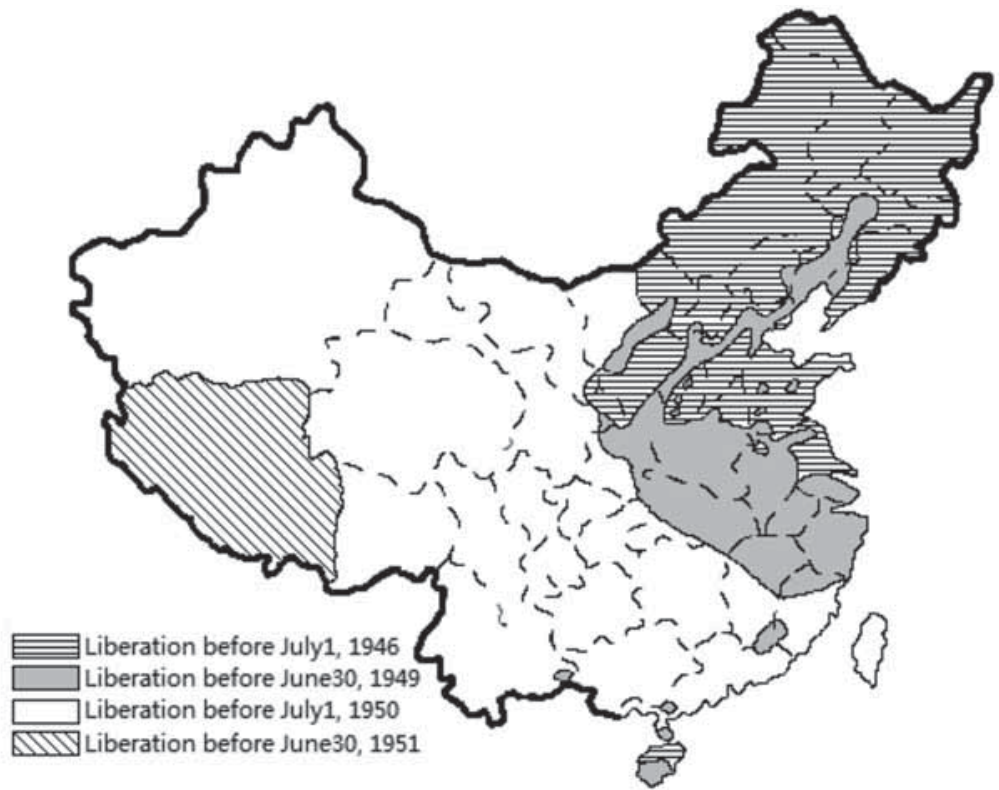

Fuente: elaboración propia a partir de China Map Press, 1995.

\subsection{División administrativa 1949-1978}

Según Dai (1999) el proceso de desarrollo de la división administrativa durante el periodo 1949-1978 se puede dividir en dos fases: formación de la estructura básica de la división territorial (1949-1958) y fase de la comuna popular (1958-1978).

\subsubsection{Formación de la estructura básica de la división territorial (1949-1958)}

La división administrativa de la Republica Popular China al comienzo de esta primera fase se muestra en la figura 5 (izquierda). En ella ya se recoge la independencia de Mongolia Exterior —reconocimiento oficial por la República Popular China en 1946-, los efectos de la «Campaña de Reducción de Provincias» — puesta en marcha después de la guerra chino-japonesa- y las nuevas provincias que habían sido incorporadas (Zhang, 1994). 

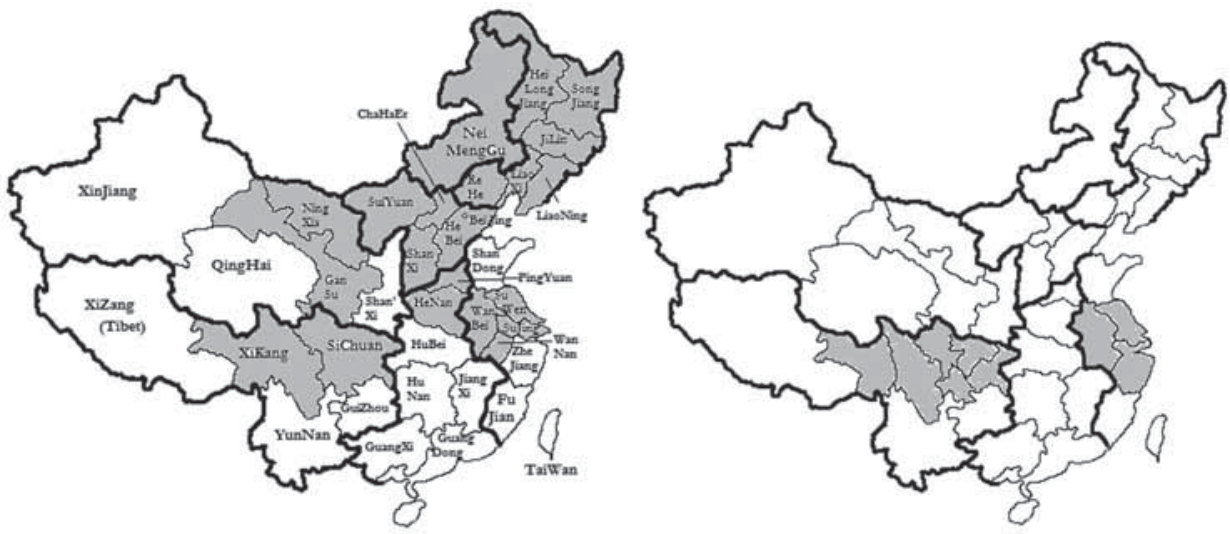

Nota: la línea gruesa dibuja el nivel de regiones administrativas. Las provincias en color gris señalan aquellas que han cambiado sus límites en comparación con el periodo anterior.

Fuente: elaboración propia a partir de China Map Press, 2009.

Desde 1949 hasta la primera Constitución (1954), las modificaciones administrativas fueron constantes con el objetivo principal de establecer el nuevo sistema administrativo, en el que se estableció como tarea principal la consolidación de la gobernabilidad local a todos los niveles (Chen y Zhang, 2009).

Así, en el año 1949, a fin de facilitar la dirección y la gestión en todo el país, el Gobierno Central estableció seis grandes regiones administrativas en el primer nivel de administración. El nivel de provincia se convirtió en el segundo, y se redujo su número (Dong y Liu, 2007) (figura 5, derecha). Hasta finales de 1952 el país estaba dividido en 54 órganos a nivel provincial.

El establecimiento de grandes regiones administrativas era ventajoso para la dirección unificada del poder político central bajo condiciones de guerra y en el corto plazo. Pero en época de paz y a largo plazo era fácil que derivase a situaciones que invitasen al separatismo. Por consiguiente, el Gobierno Central revocó las grandes regiones administrativas en 1954, a la vez que unificó algunas provincias de pequeña escala (figura 6). Esta acción redujo tanto el nivel administrativo como los gastos de gestión. Todas las municipalidades excepto Beijing, Tianjin y Shanghai se delegaron a la jurisdicción provincial. De esta manera el número de órganos a nivel provincial se redujo a 29 en 1959 (Dong y Liu, 2007) (cuadro 1). 


\section{CAMBIOS EN LA DIVISIÓN ADMINISTRATIVA EN 1953 Y EN 1954}
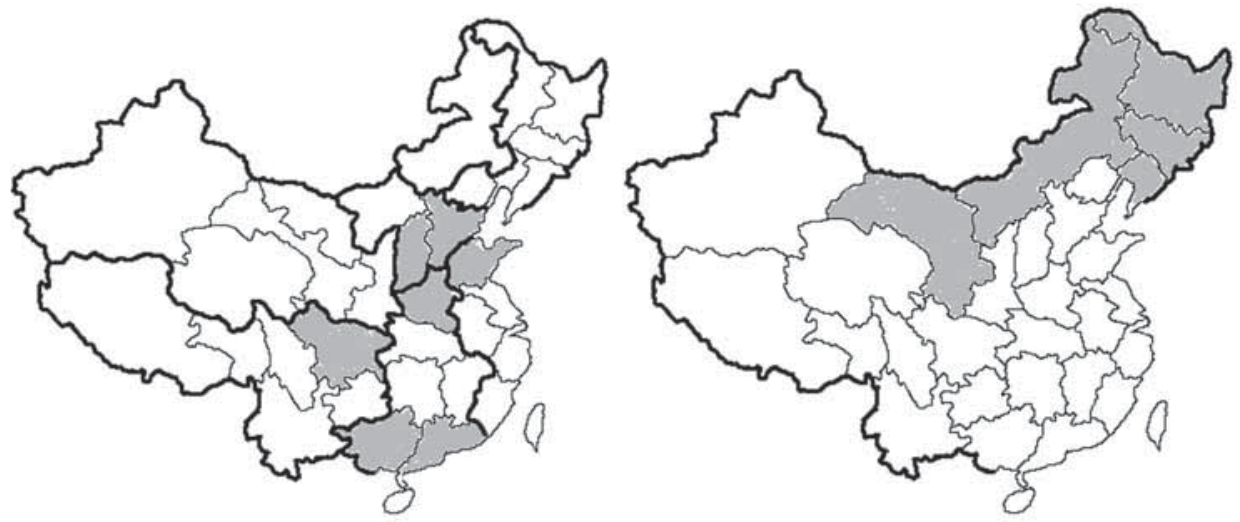

Nota: las regiones administrativas fueron derogadas en 1954. Las provincias en color gris señalan aquellas que han cambiado sus límites en comparación con el periodo anterior.

Fuente: elaboración propia a partir de China Map Press, 2009.

En la fase de fundación de China, las zonas urbanas y rurales eran administradas por separado. El sistema urbano comprendía la municipalidad, la ciudad de nivel local, la ciudad de nivel de condado, los distritos y las ciudades menores (towns). Todos los gobiernos de las ciudades gestionaban principalmente la producción industrial. Otros órganos administrativos en el nivel de provincia fueron los gobiernos rurales que incluían la oficina de representación del gobierno provincial (PGRO), el condado, la oficina distrital y las villas o pueblos; todos ellos encargados de la gestión de la producción agrícola. En algunos casos la PGRO y la ciudad a nivel local se ubicaban en el mismo lugar, y también algunos condados y ciudades a nivel de condado. En estos casos ninguno tenía el control del otro y eran unidades al mismo nivel administrativo. Pero aparecieron algunos casos donde la ciudad «lideraba» al condado (City Leads County), con el objetivo de resolver los problemas de abastecimiento de alimentos básicos que afectaban a algunas de las grandes ciudades (Hua, 2003). El esquema básico de esta división administrativa de China se muestra en la figura 7.

En el sistema administrativo de China, el condado es el nivel administrativo más antiguo y estable. Iniciado por primera vez en el periodo de Primavera y Otoño (770-476 a. C.) su base original se mantuvo con algunos ajustes moderados en este periodo. El primer aspecto que se trató en este nivel fue regular los condados que tenían un tamaño muy grande o muy pequeño, bien a 
FIGURA 7

ESQUEMA DE LA DIVISIÓN ADMINISTRATIVA CHINA

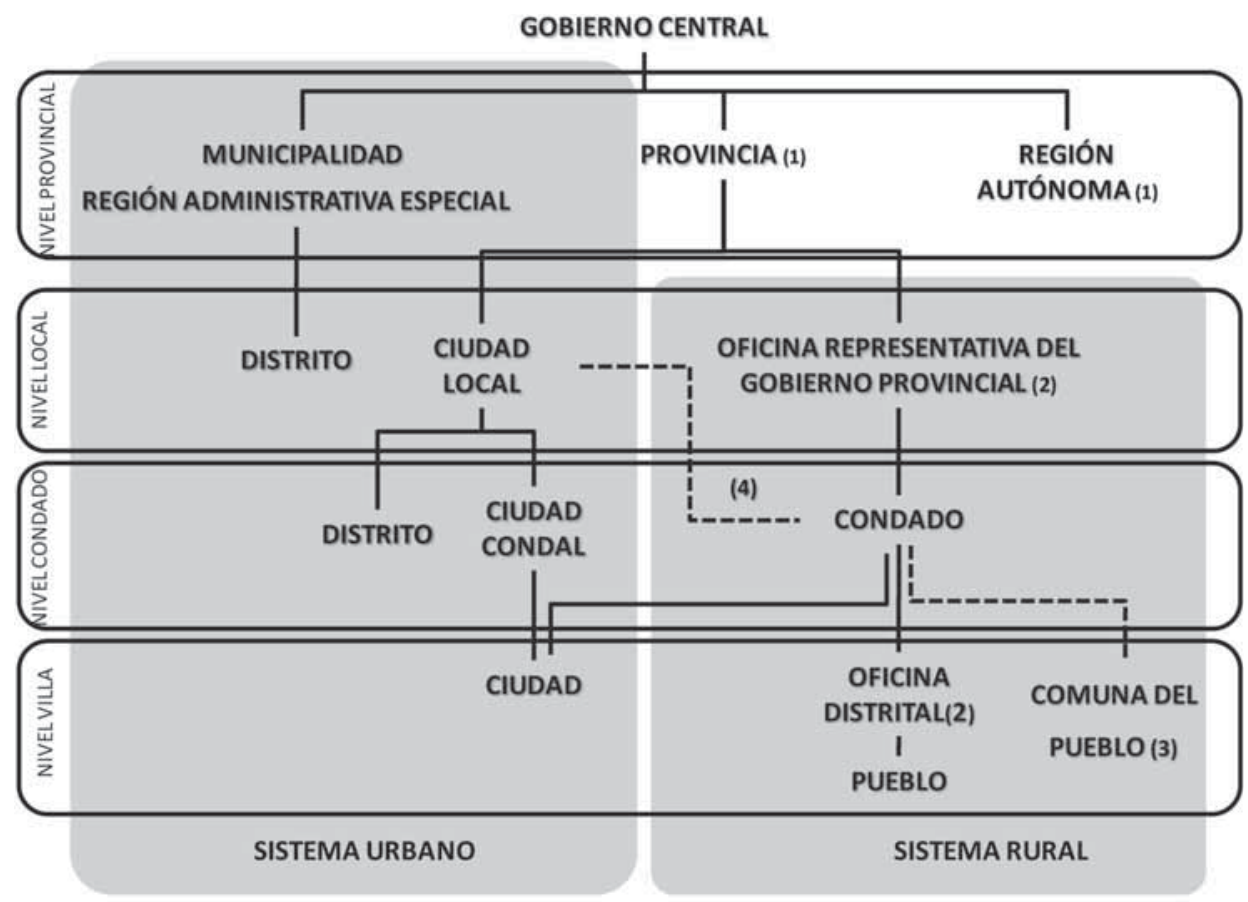

Notas: (1) El sistema autónomo no se detalla pero su estructura es similar a la provincial; (2) Oficinas desconcentradas del nivel superior; (3) la oficina distrital y el pueblo fueron sustituidos por la comuna durante el periodo de la Revolución Cultural (1966-1976), y recuperados en 1982; (4) el sistema de ciudades liderando condados fue implementado para reducir las oficinas representativas del gobierno provincial.

Fuente: elaboración propia a partir de Constitution of People's Republic of China, 1954-1982; Hua, 2003.

causa de la guerra u otras razones históricas. El segundo aspecto fue crear algunos condados nuevos dada la necesidad de desarrollo económico. Otra revisión fue la estandarización de la reforma. Así, todos los consejos que se habían establecido durante el período de la República de China en las áreas minoritarias - y que de hecho funcionaban como condado-, se transformaron de manera efectiva en condados (Dai, 1999; Zhang, 1999).

El establecimiento del gobierno popular a nivel de los pueblos o villas se inició en 1951. El Gobierno Central exigió disminuir el tamaño de la división administrativa a este nivel con el fin de facilitar la gestión por parte de la población, asegurar una relación estrecha entre el gobierno y el pueblo, y mos- 
trar claramente el nivel básico de la función de organización del gobierno popular, así como mejorar la eficiencia en el trabajo (Government Administration Council, 1951). Siguiendo esta orden cada provincia disminuyó el tamaño de los pueblos y las pequeñas ciudades en general, tomando la población como estándar, cada uno tenía alrededor de 400 hogares. Después de que los órganos a nivel de pueblo o villa se hicieran más pequeños, cada condado pasó a gestionar en promedio cerca de 100 pueblos. Para aliviar las tareas administrativas del condado, se crearon las oficinas de distrito como representaciones del condado para gobernar varias aldeas. Hasta 1953 había alrededor de 18.900 oficinas de distrito en todo el país.

Sin embargo, el incremento de las organizaciones administrativas también aumentó el coste de la gestión y la carga para la nación, por lo que en 1953 comenzó el desmantelamiento y la fusión de pueblos a gran escala. En 1954, los órganos a nivel de pueblo se redujeron aproximadamente hasta los 218.700, que era aproximadamente el equivalente al nivel de 1951.

De acuerdo a la reglamentación de la Constitución de 1954 y con el fin de satisfacer la necesidad de adaptarse al rápido desarrollo de la cooperación agrícola, el Estado abogó porque cada pueblo o ciudad se ampliará a más de 900 familias en promedio. Así, desde finales de 1955, diversas regiones comenzaron a derogar las oficinas de distrito y a unir a los pueblos. A la vez que se daba este proceso, el número de condados y PGRO también disminuyó (Dai, 1999; Liu, 2006) (cuadro 1).

La primera Constitución también reguló que la división administrativa debía tener tres niveles: la provincia, el condado y el pueblo (CPRC, 1954). Sin embargo debido a que la dimensión de las provincias era en general grande, había dificultades para que el comité de nivel provincial gobernara directamente el condado por lo que la PGRO y la gran ciudad funcionaron como nivel local. Este sistema existía antes de la fundación de la República Popular China y sigue estando vigente en la actualidad (Dai, 1999). En este periodo, el sistema urbano experimentó una etapa de desarrollo estable, situación que se refleja en las palabras de Mao en 1949:

«A partir de ahora, ha comenzado el tiempo del paso del campo a la ciudad y de que la ciudad lidere el campo, el enfoque de trabajo del Partido ha cambiado del campo a la ciudad $[\ldots]$ Tenemos que poner esfuerzos inmensos para aprender a manejar y construir la ciudad» ${ }^{1}$.

\footnotetext{
${ }^{1}$ CPC News (1949, March 5): Second Plenary Session of the Seventh Central Committee. Disponible en: http://dangshi.people.com.cn/GB/165617/index.html (Fecha de consulta: 25/01/2012).
} 
Bajo la instrucción de estos pensamientos, el Estado estableció el gobierno popular en las ciudades inmediatamente después de terminar la guerra, y promovió la restauración del orden urbano y la economía. Asimismo, con el fin de satisfacer las necesidades de desarrollo de la industria, el comercio, la minería y otras industrias, el Estado fundó numerosas ciudades nuevas (Dai, 2000) (cuadro 1).

\subsubsection{Fase de la comuna popular (1958-1978)}

Los veinte años de la segunda fase comenzaron en 1958 a partir del «Movimiento para Formar Comunas del Pueblo» y terminaron con la implementación de la «Política de Reforma y Apertura» en 1978. Los cambios en esta fase implicaron la transformación radical del nivel de pueblos y ajustes parciales en otros niveles. La mayoría de los cambios se vieron afectados por las políticas del «Gran Salto Adelante» y las campañas de la «Revolución Cultural» (Dai, 1999).

En julio de 1969, el área de la Región Autónoma de Mongolia Interior sufrió un cambio significativo. En ese momento, las relaciones entre China y la Unión Soviética atravesaban por una fase de grave tensión. Por miedo a una potencial guerra y para fortalecer la preparación de Mongolia, muchos de estos territorios se incorporaron en las provincias vecinas, por lo que la frontera nacional en Mongolia sufrió una reducción significativa (figura 8) (Galán, 1977; Yu, 1998).

\section{FIGURA 8}

\section{CAMBIOS EN LA DIVISIÓN ADMINISTRATIVA EN 1964 Y 1978}
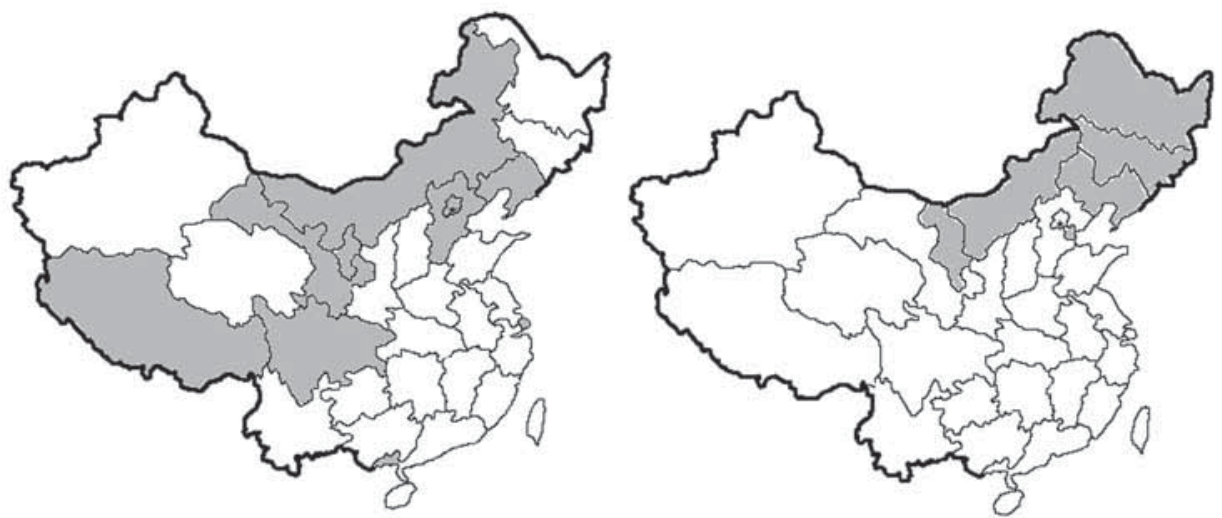

Nota: las provincias en color gris señalan aquellas que han cambiado sus límites en comparación con el periodo anterior.

Fuente: elaboración propia a partir de China Map Press, 2009. 
Para satisfacer la necesidad de desarrollo de la cooperación agrícola y el desarrollo económico colectivo (Galán, 1977), se inició en 1958 el «Movimiento de Formación de Comunas Populares Rurales» y todos los pueblos y las oficinas de distrito fueron cambiados para uniformizar las comunas. La comuna popular es la unidad básica de la combinación de los trabajadores, los campesinos, los comerciantes, los estudiantes y los soldados en la estructura social socialista; también es la unidad básica de organización política socialista. De acuerdo con un estándar de gran tamaño y de naturaleza colectiva, una comuna popular tenía unos 6.000 ó 7.000 hogares, aunque en algunos casos llegaron incluso a los 20.000 (Liu, 2006; Resolutions on People's Communes, 1958). A finales de 1958, la nación en conjunto había creado 26.578 comunas populares, las cuales eran igual al tamaño de tres municipios originales, como promedio. Dicho tamaño, obviamente, era grande para las condiciones del transporte y de las comunicaciones en ese momento, por lo que se ajustó gradualmente (Dai, 1999) (cuadro 1).

Por otro lado Mao siempre dio gran importancia al desarrollo industrial (Ba, 1993) y en 1958 copió el método japonés de creación de industrias en las zonas rurales, comenzando a desarrollar la industria en los niveles provincial, local y de condado. Animados también por los grandes objetivos y el alarde del «Gran Salto Adelante», se iniciaron proyectos industriales en la mayor parte de las 183 grandes ciudades y en las más de 2.000 pequeñas ciudades que se repartían por todo el país. Bajo este esquema, se suponía que ciudad e industrialización eran lo mismo, lo que consecuentemente condujo al establecimiento de muchas ciudades nuevas (Dai, 1999 y 2000). Al mismo tiempo los programas industriales se estaban realizando también en los condados. En este proceso de cambio los condados agrícolas se transformaron en condados industriales, a fin de lograr objetivos como facilitar la explotación conjunta de los recursos, mejorar el poder económico de los condados, etc... Un gran número de condados se unieron en este período conocido como Combine Big Counties. A medida que la campaña reducía el número de condados, las PGRO también se unieron o fueron revocadas (Zhang, 1999).

Al comienzo de este período, con el propósito de que la ciudad pudiese desempeñar un papel de liderazgo en la industrialización rural y que las zonas rurales pudiesen garantizar el abastecimiento alimentario de la ciudad, la implementación del sistema City Leads Counties se amplió y se desarrolló rápidamente (ver nota 4 de la figura 7). Así, a finales de 1960, 50 ciudades de nivel local y 2 municipios lideraban 237 condados. Sin embargo desde la época de los desastres naturales la PGRO fue restaurada de manera general y para el año 1965 quedaban solo 24 ciudades liderando 61 condados (Zheng, 2009). 


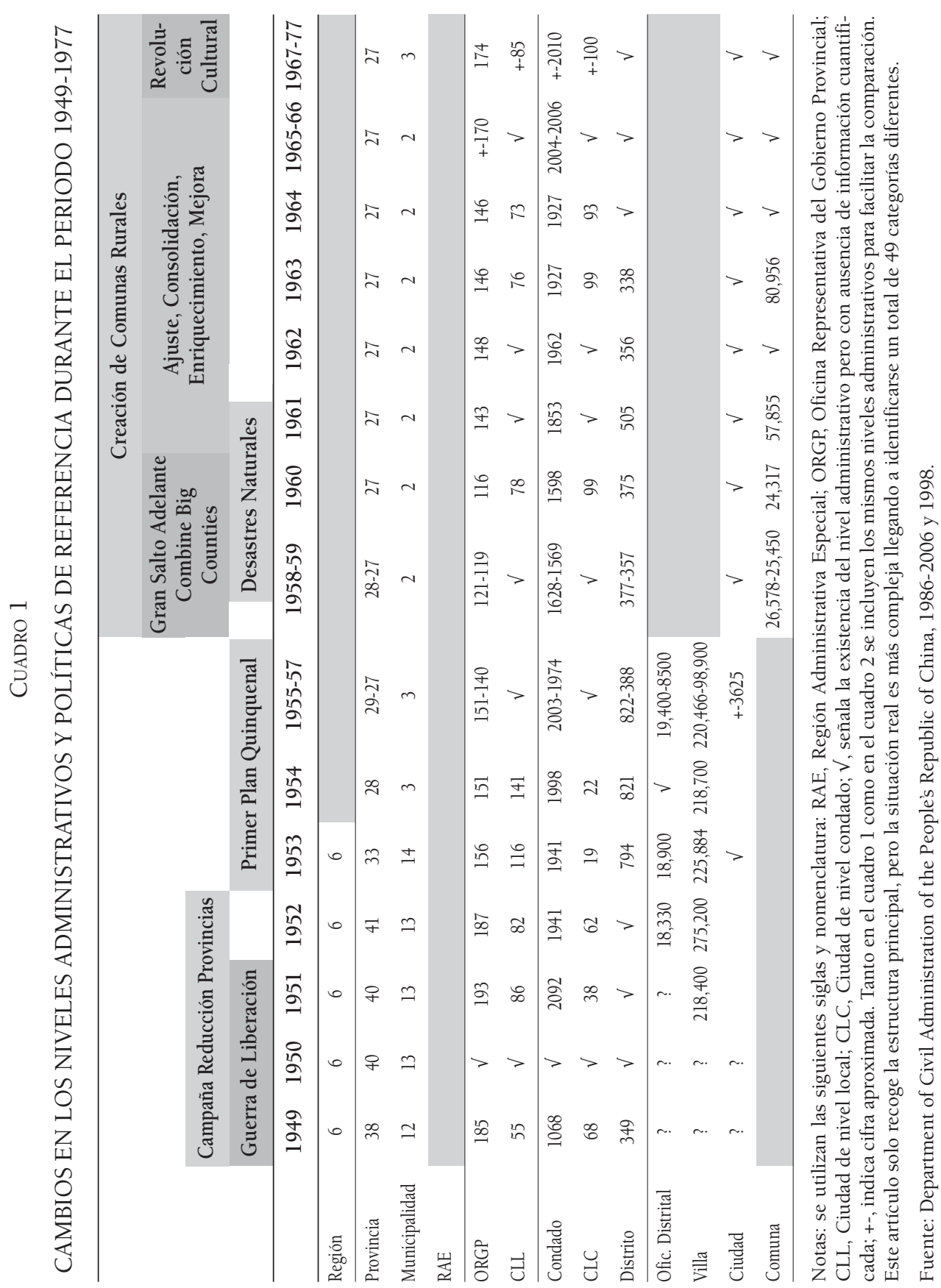

Estudios Geográficos, Vol. LXXIII, 272, pp. 273-307, enero-junio 2012 ISSN: 0014-1496, eISSN: 1988-8546, doi: 10.3989/estgeogr.201210 
Debido a la demanda industrial de trabajadores, una gran cantidad de campesinos comenzaron a trabajar en las fábricas o se mudaron hacia las ciudades y los pueblos. La alta tasa de crecimiento de la población urbana, unido a los tres años de desastres naturales (1959-1961), provocaron que la demanda de alimentos superara la capacidad de la oferta agrícola. Así, en 1961, el Comité Central llevó a cabo el principio de «ajuste, consolidación, enriquecimiento y mejora», para corregir estas dinámicas. Así, en los cinco años anteriores a la «Revolución Cultural», se adoptaron una serie de medidas para regular los proyectos industriales, para reducir la población urbana y para revocar ciudades y pueblos no calificados. En 1965 el número de ciudades y pueblos disminuyeron, los condados superaron los 2.000 y las PGRO fueron reinstauradas y conocieron un incrementado de su número (Dai, 1999 y 2000) (cuadro 1).

Con la «Revolución Cultural» (1966-1976) la economía nacional sufrió graves daños, especialmente en los aspectos industriales, cuya fluctuación limitó en gran medida el desarrollo de las ciudades. A la vez la tendencia ultraideológica influyó en la no construcción de zonas urbanas. Todas las fábricas que se levantaron entre 1966 y 1971 se encuentran en las zonas de montaña (Dai, 2000). En conjunto el número de ciudades fluctuó durante el período de la «Revolución Cultural», si bien en este aspecto la ausencia de información es palpable. Destacar también que durante el período de la Revolución Cultural la PGRO se constituyó como un nivel oficial de acuerdo con la Constitución de 1975, por lo que el sistema administrativo se convirtió oficialmente en uno de cuatro niveles (Constitution of People's Republic of China, 1975; Dai, 1999; Chen y Zhang, 2009).

\subsection{Desarrollo regional equilibrado}

En este período el desarrollo de China se basó en el principio de equidad. Las políticas del Gobierno trataron de eliminar las diferencias en el ingreso y en el crecimiento entre los grupos de población, las clases y las regiones.

En particular cabe destacar como el desarrollo de las ciudades costeras de China se estancó durante este período por dos razones. En primer lugar, China tenía limitada interacción económica con el exterior. Durante la era de la Guerra Fría, el comercio exterior de China se limitó al interior del bloque socialista soviético, además, se vivieron situaciones de gran tensión entre los comunistas chinos y los nacionalistas, especialmente a lo largo del estrecho de Taiwan. Asimismo, después de la ruptura con la Unión Soviética a inicios de la década de los sesenta, China mantuvo una política de puertas cerradas y 
se aisló de la economía mundial. La segunda razón es que la economía china se guió por una política de desarrollo dirigida a la industria pesada que acaparó las prioridades en la asignación de recursos a expensas de las industrias de la luz y los sectores del comercio y de las finanzas. La política de la industria pesada fue especialmente desalentadora para el crecimiento de las ciudades costeras, como el comercio, las finanzas y el entretenimiento fueron los principales sectores económicos de este último (Sun y Yan, 1999; Gernet, 2006 y 2008).

En conjunto, durante los treinta años que se desarrollan entre 1949 y 1978, China estuvo explorando constantemente un sistema territorial más razonable para una administración eficiente. La categoría y la cantidad de los órganos administrativos fueron ajustadas continuamente para lograr este propósito. Aunque se cometieron errores, podemos decir que se fue acumulando experiencia para conseguir orientar el desarrollo en el período siguiente.

\section{3. Época de la Reforma y de Apertura}

El segundo período analizado se desarrolla desde 1978 hasta la actualidad, durante el cual China está experimentando un cambio gradual desde una sociedad rural, austera y autosuficiente hacia una sociedad urbana, de consumo y más abierta (Fabre, 2002). Con moderados ajustes en la división administrativa y con un núcleo importante de actuaciones en cuanto a la descentralización administrativa el sistema de responsabilidad fiscal, el sistema de división de los impuestos y el sistema de personal, este período implica una descentralización multi-nivel del sistema administrativo, (Chen y Zhang, 2009).

Cuando Mao muere en 1976, Deng Xiaoping se convierte en el líder supremo de China. Deng aplicó la política de «Reforma y Apertura» en 1978, desde entonces, el enfoque de trabajo del Partido pasó de la lucha de clases a la construcción económica como tarea principal (Fan, 1997). En 1979 el Gobierno Central, Guangdong y la provincia de Fujian decidieron experimentar la política de «Zona Económica Especial» con el propósito de implementar políticas especiales y medidas flexibles en las actividades económicas de cara al exterior. En 1984 China cambió el sistema social de una economía de planificación socialista a un camino de desarrollo de la economía socialista de mercado. Hong Kong fue reintegrado a China por Gran Bretaña en 1997 y Macao por Portugal en 1999, lo que puso en práctica el concepto de Deng «Un País, Dos Sistemas» (Editorial Committee on Party Literature of the Central Committee, 1993). En 1999, el Comité Central anunció la nueva po- 
lítica de «Desarrollo Occidental» para impulsar el crecimiento de esta región, lo que también simbolizaba que China comenzaba a luchar contra las diferencias de desarrollo cada vez mayores entre sus regiones. El impuesto agrícola fue abolido en todo el país en 2006, reduciendo la carga de los campesinos y tratando de disminuir la disparidad entre las zonas urbanas y las rurales (Guo, 2007; Roux, 2006).

La República Popular China terminó así con el periodo de aislamiento y el desarrollo económico ineficiente de Mao. La política de Deng reintrodujo a China en las relaciones internacionales y logró un rápido crecimiento económico. Pero la política de desarrollo desigual de Deng también dio lugar a una creciente diferencia de desarrollo entre las zonas costeras y las del interior. El Séptimo Plan Quinquenal se hacía eco de esta situación y recomendaba que las inversiones en la zona del interior aumentaran una vez más (Fan, 1997).

\subsection{División administrativa}

En este período hay dos cambios principales de la división administrativa. El primero es la transformación de la comuna popular. El segundo consistió en fusionar la PGRO con la ciudad con la que compartía nivel, o bien transformarla en ciudad. Este último proceso comenzó en 1982 y ha continuado hasta el presente (Dai, 1999).

Por otra parte se realizaron nuevos cambios administrativos en el nivel de la provincia: en 1979 fue devuelta a la Región Autónoma de Mongolia Interior la parte del territorio que había sido incorporada a otras provincias ( $\mathrm{Yu}$, 1998); con el fin de mejorar el desarrollo de la región costera de China fue establecida en 1984 la provincia de Hainan; el municipio de Chongqing fue creado en 1997 para acelerar la exploración y el desarrollo del oeste de China y finalmente, tal y como se ha referido, se establecieron las regiones administrativas especiales de Macao y Hong Kong en 1997 y 1999 (Qu y Chai, 2009). El resto de la división administrativa de China a nivel provincial se mantiene sin cambios (figura 9).

La política de «Reforma y Apertura» partía de la innovación del sistema económico en el área rural, que fue la aplicación del «Sistema de Contrato con Remuneración Ligada a los Resultados». Sustituía a la comuna popular, que encarnaba la unidad de política y economía, y que restringía el entusiasmo productivo y la iniciativa de los campesinos (Constitution of People's Republic of China, 1982; CPC Central Committee - State Council, 1983a y 1983b). 
FIGURA 9

DIVISIÓN ADMINISTRATIVA DESDE 1999 HASTA LA ACTUALIDAD

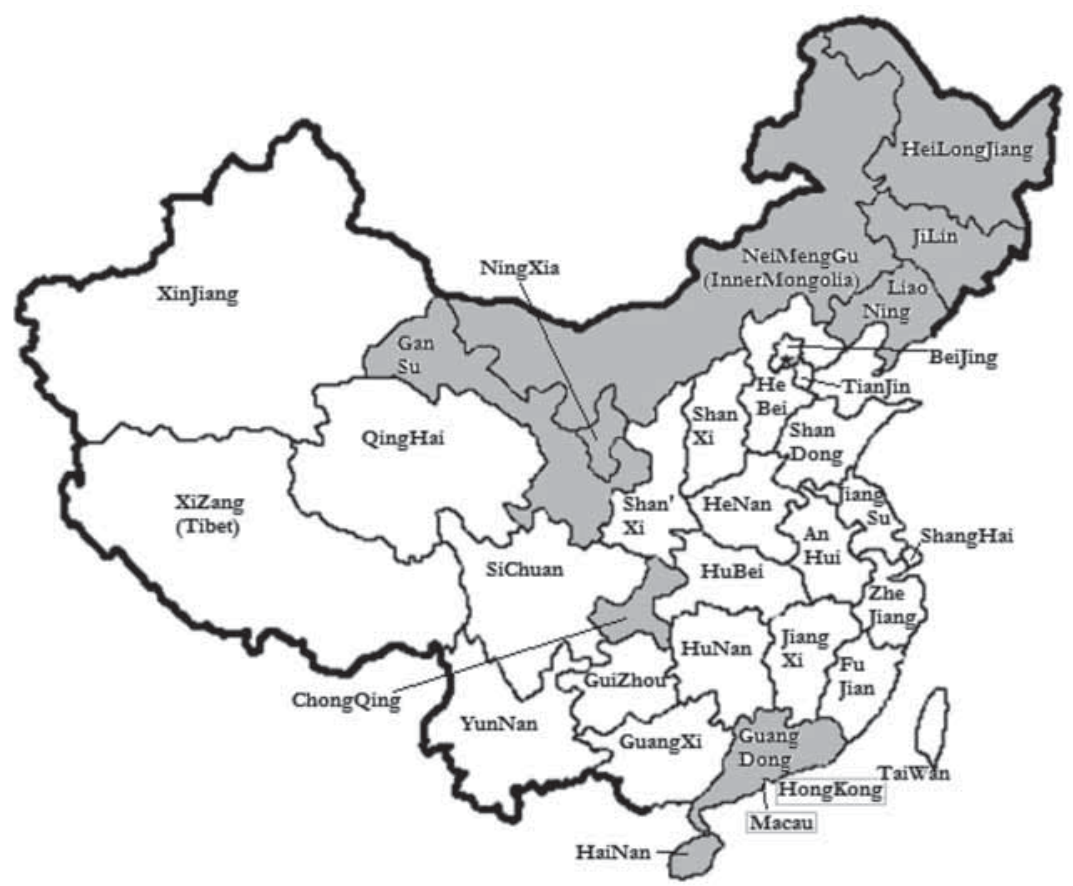

Nota: Las provincias en color gris señalan aquellas que han cambiado sus límites en comparación con el periodo anterior.

Fuente: elaboración propia a partir de China Map Press, 2009.

De este modo la eliminación de las comunas y la construcción de pueblos avanzó en toda la nación. En este proceso, el tamaño de los pueblos disminuyó y las oficinas de distrito se reinstauraron. El número de pueblos llegó a 85.200 y surgieron más de 8.100 oficinas de distrito. Después de un año de operación, muchos pueblos en la mayoría de los lugares eran demasiado pequeños, por lo que comenzó de nuevo un proceso de cancelación de oficinas de distrito y de unión de pueblos. A finales del año 1986, estos últimos habían disminuido a cerca de 58.400. En el procedimiento de cancelación de las comunas, en los lugares con buenas condiciones económicas se convirtieron en pequeñas ciudades, por lo que su número se elevó de 2.173 en 1978, a 20.600 en 2002 (Dai, 1999; Liu, 2006) (cuadro 2). 


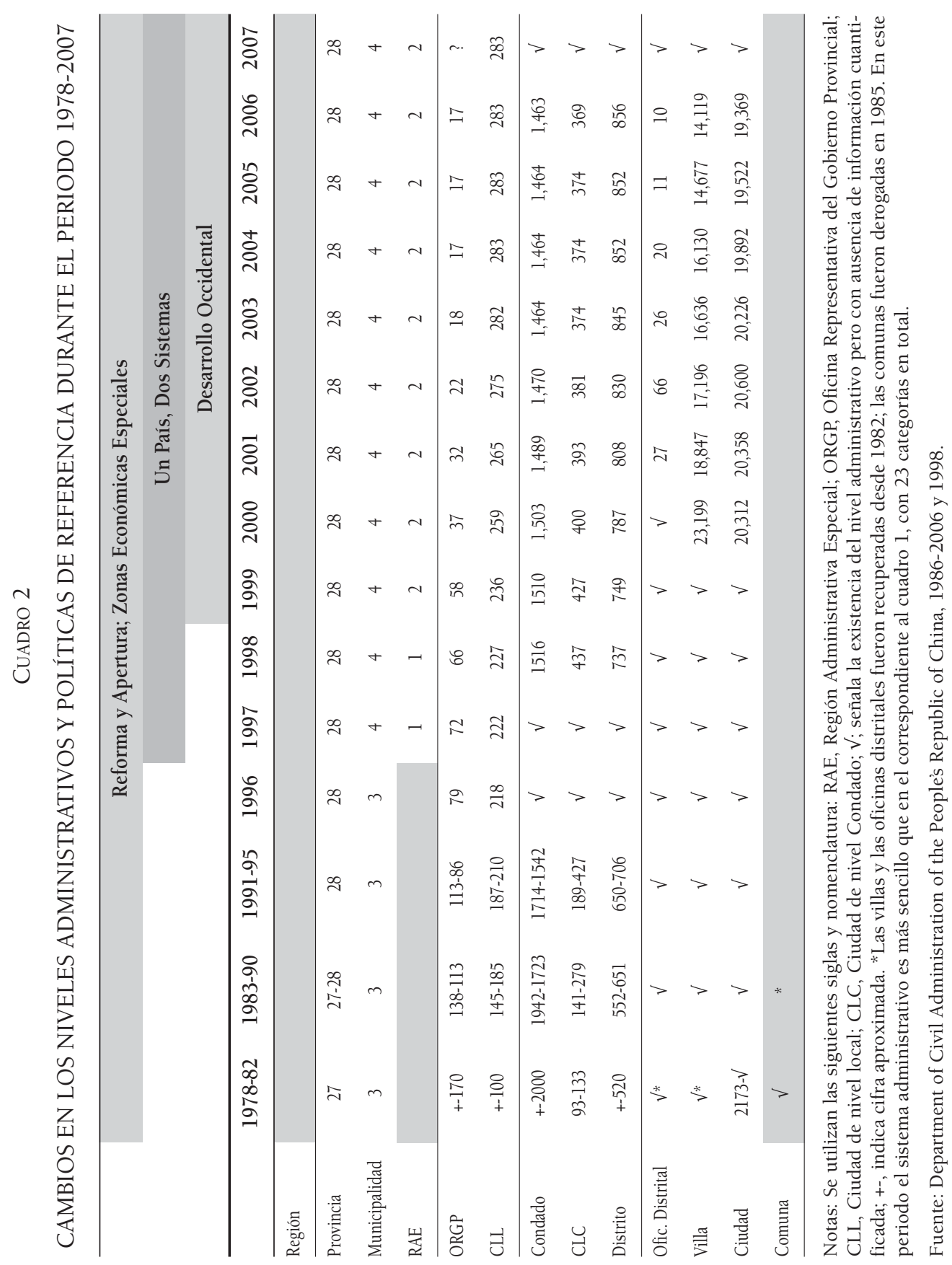

Estudios Geográficos, Vol. LXXIII, 272, pp. 273-307, enero-junio 2012

ISSN: 0014-1496, eISSN: 1988-8546, doi: 10.3989/estgeogr.201210 
Junto con el avance de la «Reforma y Apertura» el conflicto que suponía que la PGRO y la ciudad se ubicasen en el mismo lugar fue en aumento, el sistema de gestión trabajó con muchos contratiempos y muchos gastos administrativos en vano. Las notificaciones de 1982 y de 1983 indicaron que la siguiente reforma debería tomar las ciudades económicamente desarrolladas como el centro y la vasta zona rural como base para desarrollar el sistema City Leads County gradualmente, con el fin de combinar fuertemente las zonas urbanas y rurales (Zheng, 2009). Esta reforma tuvo dos modelos principales: en el caso de que la PGRO residiese en una ciudad a nivel local, se combinaba con esta última manteniendo la ciudad; en el caso de que la PGRO residiese en una ciudad de nivel de condado ambas eran suprimidas para formar una ciudad a nivel local (State Council, 1986; Dai, 1999).

Según los datos disponibles de 2001, 153 de las 265 ciudades a nivel local dirigían 1.445 condados. Desde la reforma iniciada en 1982 el número de PGRO se redujo en gran medida de 170 a 17 en 2006 (Li y Deng, 2009; Zheng, 2009). Esta reforma explica en parte por qué el número de ciudades a nivel de condado se redujo desde 1998. Al mismo tiempo, una gran cantidad de ciudades a nivel de condado fueron ajustadas a distritos de ciudades de nivel local, lo que también redujo el número de ciudades a nivel de condado (Qu y Chai, 2009).

Pero junto con el desarrollo de la comercialización, la industrialización y las empresas del mundo de la información, las desventajas del sistema City Leads County llegaron a ser muy importantes. Este sistema reducía el poder de decisión a nivel de los condados, pero incrementó las diversas autoridades administrativas de las ciudades del nivel local. En algunas regiones existía el fenómeno denominado como «Pequeños Caballos Arrastrando Grandes Carros» por el cual las ciudades a nivel local no son capaces de irradiar y estimular el desarrollo económico del condado. Por lo tanto, varios lugares comenzaron a explorar un nuevo sistema de Province Leads County. En 2009, el Gobierno Central comienza a impulsar el sistema de finanzas de este nuevo enfoque, avanza con la reforma experimental de «Expandir un Poder Fuerte del Condado», promueve las provincias con buenas condiciones para reducir los niveles administrativos y explora legalmente el sistema Province Leads County (Li y Deng, 2009; Ministry of Finance, 2009).

\subsection{Estrategias para el Desarrollo Regional}

Desde la «Reforma y Apertura» las tendencias del desarrollo regional pueden resumirse en dos estrategias: por un lado la estrategia de prioridad hacia la costa y 
por otro, la lucha contra la brecha creciente entre la China oriental y la occidental y entre las regiones urbanas y las rurales (Feng, Wu y Zhu, 2008; Fabre, 2002).

\subsubsection{Estrategia de prioridad hacia la Costa}

Después de 1978 se acusó al excesivo énfasis por la equidad de ser un obstáculo contra el desarrollo económico. Por lo tanto el Gobierno Central adoptó una política para permitir que algunas personas y algunas regiones pudiesen enriquecerse por encima de la media (Sun y Yan, 1999). China desplazó el énfasis de ubicación del desarrollo a la región costera, a ciudades que proporcionaban una buena conexión con la economía mundial, fácil acceso a los recursos marinos y mejores condiciones económicas. A finales de la década de 1970 el Gobierno estableció cuatro zonas económicas especiales a lo

FIGURA 10

\section{LOCALIZACIÓN DE LAS CUATRO ZONAS ECONÓMICAS ESPECIALES Y LAS CATORCE CIUDADES COSTERAS ABIERTAS}

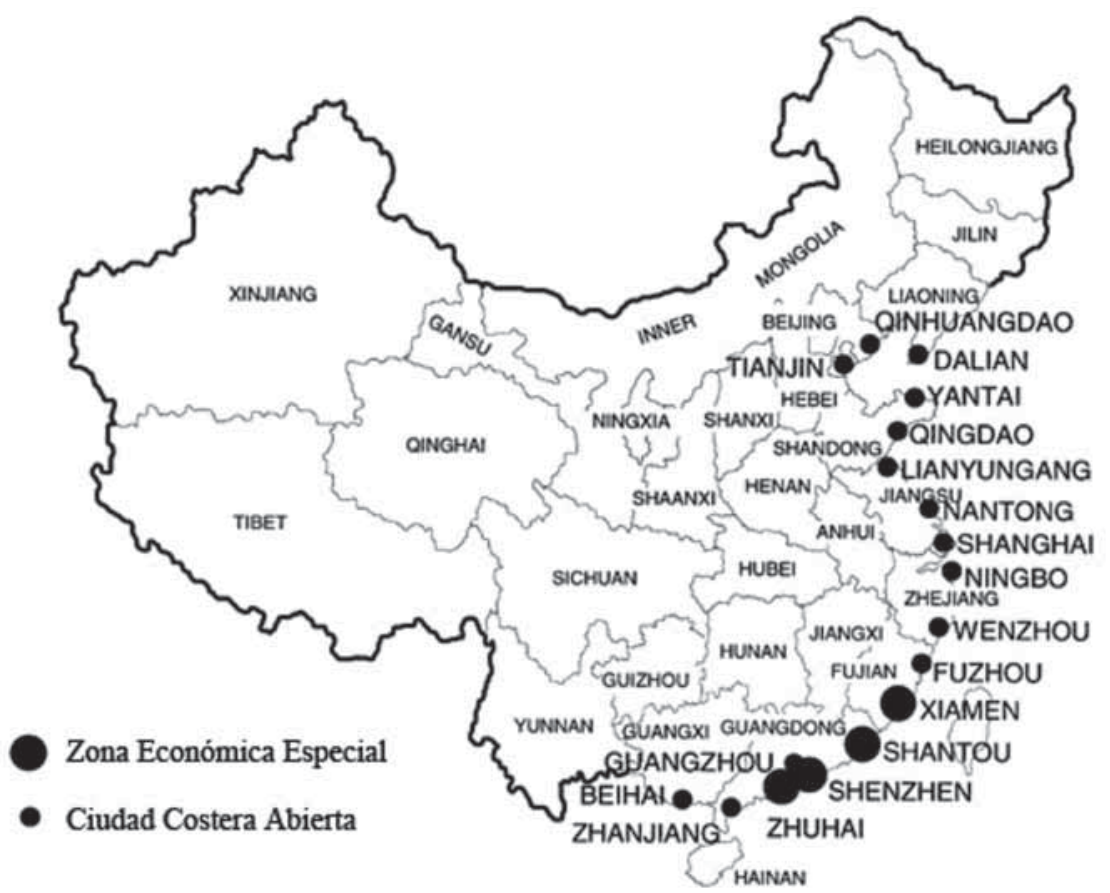

Fuente: Sun and Yan, 1999.

Estudios Geográficos, Vol. LXXIII, 272, pp. 273-307, enero-junio 2012

ISSN: 0014-1496, eISSN: 1988-8546, doi: 10.3989/estgeogr.201210 
largo de la costa de Guangdong y Fujian (Han y Wong, 1994). A principios de 1980, a 14 ciudades de la costa les fue concedido el estatuto de "Ciudades Costeras Abiertas» (figura 10). Las especiales políticas favorables dadas a estas ciudades, incluyendo un menor control del Gobierno Central e incentivos a la inversión, han llevado a un rápido desarrollo a lo largo de toda la costa y desde entonces han actuado como motores del desarrollo económico (Yeung y Hu, 1992; Fan, 1995; Lin, 1995; World Bank, 1997).

Por otro lado, en la Ley de Planificación Urbana aprobada por el Consejo de Estado en 1989 (Ministry of Construction City Planning Division, 1990), se requiere a los gobiernos de los distintos niveles tener un plan regional para el desarrollo de las ciudades y de los pueblos dentro de su área de jurisdicción. Los ejemplos de planificación del sistema urbano incluyen las «Estrategias de Desarrollo Urbano alrededor del mar de Bohai» y el «Plan del Sistema Urbano en el delta del río Perla». Estos documentos han servido a las ciudades como directrices para políticas integradas en la formulación de sus estrategias y en el diseño de sus planes de desarrollo. También han jugado un papel importante en la coordinación de los programas regionales. Las ciudades costeras también comenzaron a hacer hincapié en las variedades de la planificación del uso del suelo zonificando en áreas de la costa propicias para el turismo, como áreas recreativas o parques, a la vez que aquellas con buenas condiciones portuarias se zonificaban para puertos y embarcaderos (Sun y Yan, 1999).

\subsubsection{Combatiendo la ampliación de la brecha de desarrollo}

En el Séptimo Plan Quinquenal (1986-1990) China propuso un gradiente de desarrollo económico regional y empezó por dividir el país en tres zonas económicas diferentes (figura 11). Las tres zonas económicas representan el nivel más alto de China en cuanto a división económica, presentando de forma objetiva las graduales diferencias regionales y la tendencia general del desarrollo económico regional. Además representan el cambio estratégico de China por combatir la brecha entre las regiones (China Map Press, 2009).

Al mismo tiempo que se aceleró el paso de las reformas en la región costera, también comenzó el proceso de apertura en el interior (Chen, 1999). Tomando el costo de la zona oriental como base y apoyo de la tecnología, China podría invertir los recursos centrales y mejorar el desarrollo de la región occidental (Fourth Conference of the Sixth National People's Congress, 1986/Marzo; China Map Press, 2009). 
FIGURA 11

DIVISIÓN EN TRES ZONAS ECONÓMICAS DEL SÉPTIMO PLAN QUINQUENAL

(1986-1990)

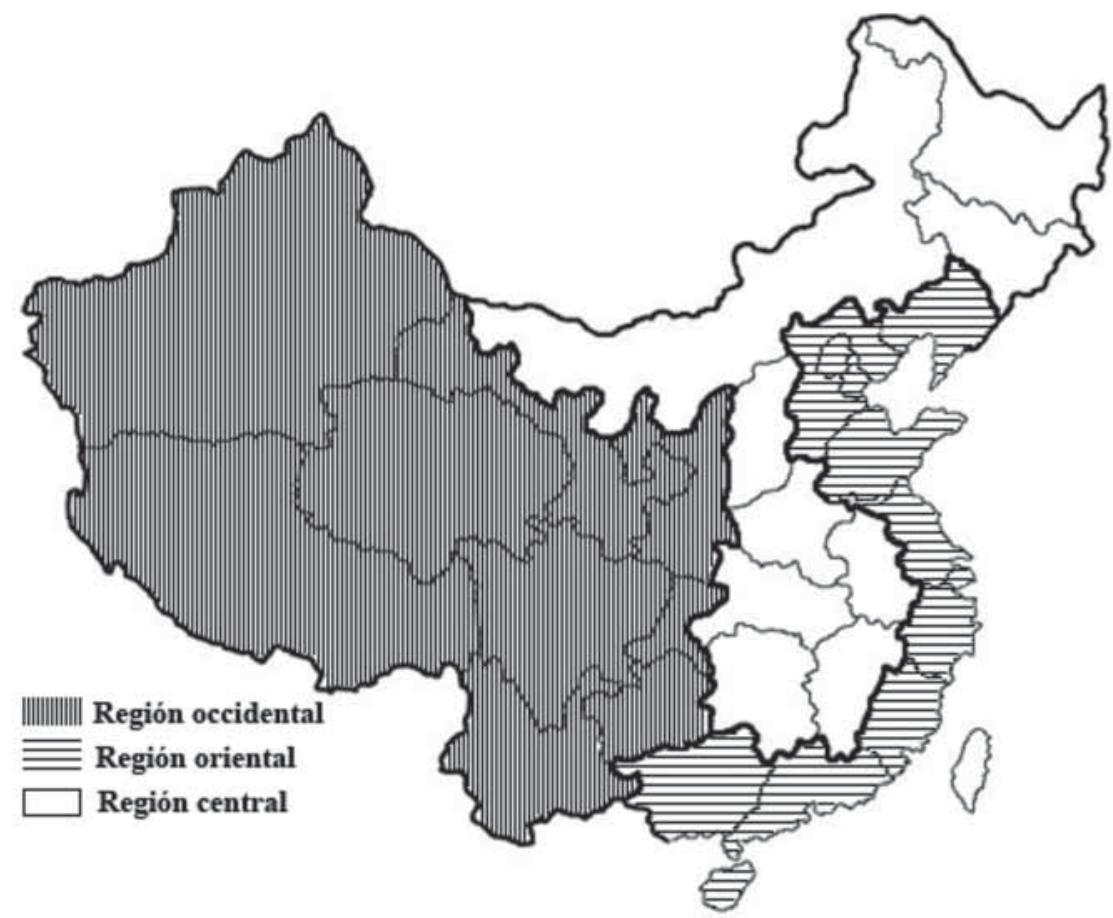

Fuente: elaboración propia a partir de China Map Press, 2009/September 16.

Mientras que las regiones costeras adquirían un crecimiento más rápido la diferencia entre la región oriental y occidental se amplió considerablemente (Fan, 2004). Sobre la base de esa situación, el Gobierno Central ajustó y mejoró constantemente la estrategia de desarrollo regional y abrió progresivamente la región del medio oeste. En 1999, el Comité Central anunció la nueva política de «Desarrollo Occidental» para impulsar el crecimiento de esta región. Se hacía hincapié en los actuales y en los futuros proyectos de desarrollo de los transportes, de las industrias y de la explotación y la transmisión de los recursos energéticos (CPC Central Committee - State Council, 1999). Hasta ahora los grandes proyectos del ferrocarril Qinghai-Tíbet, el transporte de gas natural, la distribución de electricidad del oeste al este y desvío de agua de sur a norte han avanzado sin problemas. 
FIGURA 12

REVISIÓN DE LAS TRES ZONAS ECONÓMICAS INCLUYENDO LA REGIÓN OCCIDENTAL

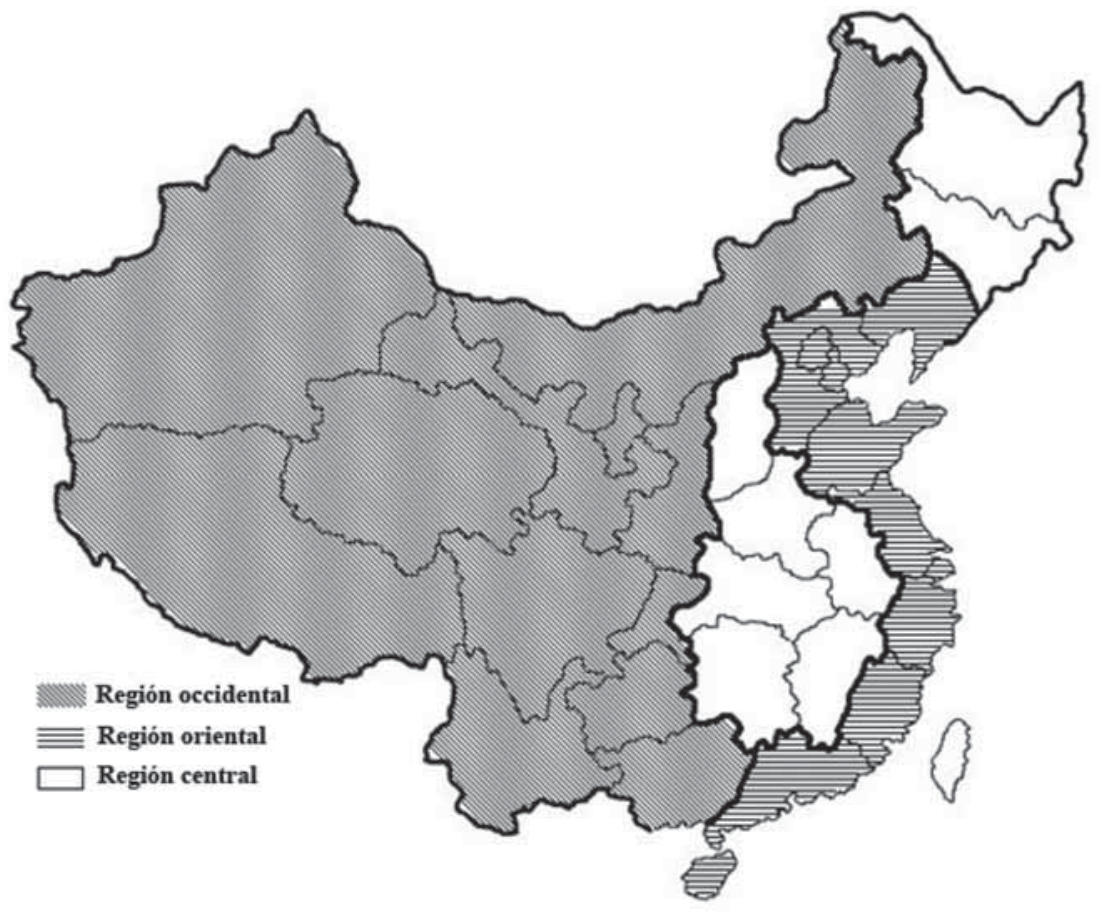

Fuente: elaboración propia a partir de CPC Central Committe \& State Council, 1999; China Map Press, 2009.

Con la propuesta de «Desarrollo Occidental», la división de las tres zonas económicas también se revisó (figura 12). Puesto que China ocupa extensos territorios, dentro de cada una de las tres zonas económicas el estado, el carácter y la situación del desarrollo económico son diferentes. En consecuencia, en el Noveno Plan Quinquenal (1996-2000) China dio un paso más para dividir a la nación en siete zonas económicas, de tal modo que cada zona pudiese desarrollarse de acuerdo con su carácter propio y su situación (Fourteenth CPC Fifth Plenary Session, 1996; China Map Press, 2009) (figura 13).

La zona I es la zona económica de Bohai: teniendo como núcleo a Beijing, se compone de estas áreas cruciales que la política nacional orienta al desarrollo. La zona II es la zona económica noreste: sus factorías de petróleo y de pro- 
FIGURA 13

SIETE ZONAS ECONÓMICAS DEFINIDAS EN EL NOVENO PLAN QUINQUENAL (1996-2000)

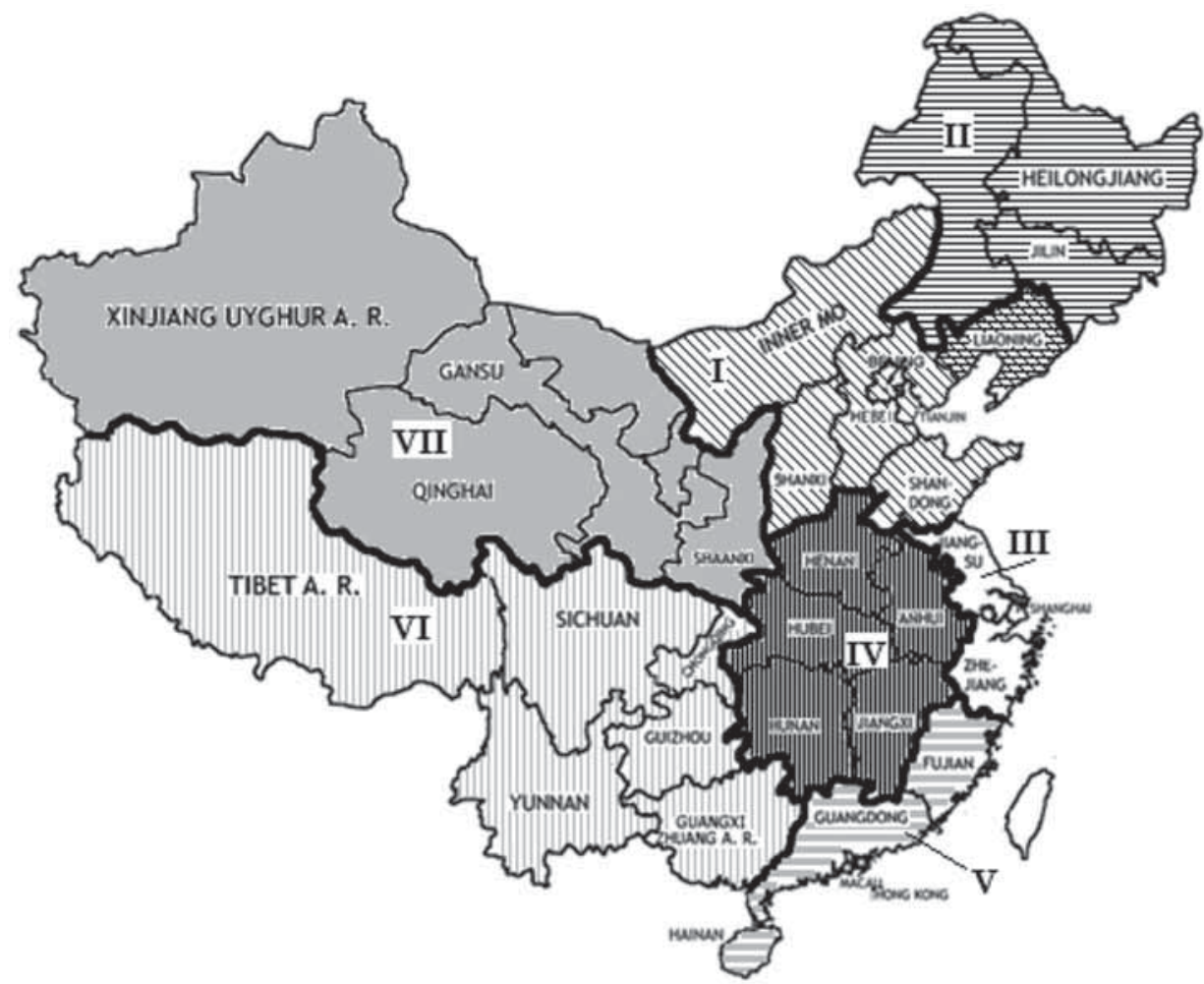

Fuente: elaboración propia a partir de China Map Press, 2009/September 16.

ductos químicos, sus equipos de minería, de electricidad y de transporte, son las de mayor importancia en el país. La zona III es la zona económica de la ribera y delta del río Yangzi y constituye el núcleo de la economía y de la tecnología en el país. Basada en la prevalencia de los recursos agrícolas locales, dominada por las industrias ligeras y textiles pero también con bastantes industrias pesadas, esta zona cuenta con un sistema integral de desarrollo económico. La zona IV es la zona económica central: es la región de transición entre las zonas económicamente desarrolladas y el oeste. Con condiciones naturales muy favorables, esta zona mantiene una importante base agrícola. La zona $\mathrm{V}$ es la zona económica de la costa sureste: es la primera línea de la polí- 
tica de «Reforma y Apertura», las zonas económicas especiales se encuentran aquí y el nivel de ingresos es el más alto de todo el país. La zona VI es la zona económica suroeste: tiene una cartera de recursos potenciales pero adolece de falta de explotación. La zona VII es la zona económica noroeste: su entorno ecológico es muy frágil pero cuenta con abundantes recursos naturales, especialmente recursos de energía y de minería. Muchas minorías nacionales viven aquí y el nivel de desarrollo económico en su conjunto es bajo (Fourteenth CPC Fifth Plenary Session, 1996; China Map Press, 2009).

En los últimos años, las tendencias en la planificación regional han cambiado desde el auto-desarrollo a la interacción multilateral; de la convergencia de la industria a la dependencia funcional; de la simple planificación de metas económicas a la planificación global de multi-objetivos y a la planificación regional integrada y el desarrollo urbano-rural coordinado (Feng, Wu y Zhu, 2008). El modelo de planificación del territorio ha pasado a organizar adecuadamente los puntos, las líneas y las áreas², y a optimizar la estructura espacial mediante una adecuada combinación de estos tres elementos (Roberts y Murray, 2002; Chang, 1997; Lu 1995).

Un ejemplo para explicar la madurez de la ordenación del territorio chino después de la política de «Reforma y Apertura» lo constituye la planificación del delta del río de la Perla en donde se pone de manifiesto el buen funcionamiento de la teoría de la gobernabilidad espacial en las aglomeraciones urbanas. La gobernabilidad es la expansión y el desarrollo de una gestión moderna que hace hincapié en la comunicación, la coordinación y la cooperación de múltiples estructuras o varios grupos con el fin de promover la cooperación y el desarrollo interregional o inter-industrial (Feng, Wu y Zhu, 2008).

El delta del río Perla es una de las primeras áreas en las que se implantó la reforma orientada al mercado en la década de 1970. Desde entonces, este espacio se ha convertido en una de las regiones más urbanizadas del país y con mayor grado de concentración de factores económicos. Aunque la competencia de la región central se ha formado y tiene su propia posición en tanto relación de producción interna e internacional y de servicios, el desarrollo acelerado de la economía urbana ha traído un gran número de problemas como el incremento de la brecha social urbano-rural, el consumo elevado de recursos, el deterioro ecológico y la competencia desordenada entre las ciudades de la región (Chun, 2006). Para resolver estos problemas, una de las solu-

\footnotetext{
2 Puntos: asentamientos, bases industriales y centros; Líneas: canales de transporte y corredores ecológicos; Áreas: zonas con ciertas funciones territoriales
} 
ciones es la gobernabilidad espacial a través de la zonificación de las políticas que se lleva a cabo en el «Plan de Desarrollo de Coordinación de Aglomeración Urbana del delta del río de la Perla (2004-2020)». El esquema consiste en dividir toda la región en nueve categorías de zonas y aplicar las políticas correspondientes, basándose en la economía, la sociedad, el medio ambiente, la industria, las condiciones de transporte de cada sitio y su impacto económico y social en el desarrollo general de toda la región (Gobierno Popular de la provincia de Guangdong, 2005) (figura 14).

Esta política regional en el delta del río Perla pretende alcanzar objetivos que incluyen el desarrollo de la economía, el progreso social, el uso sostenible de los recursos, la conservación ecológica, el desarrollo sostenible... e intenta

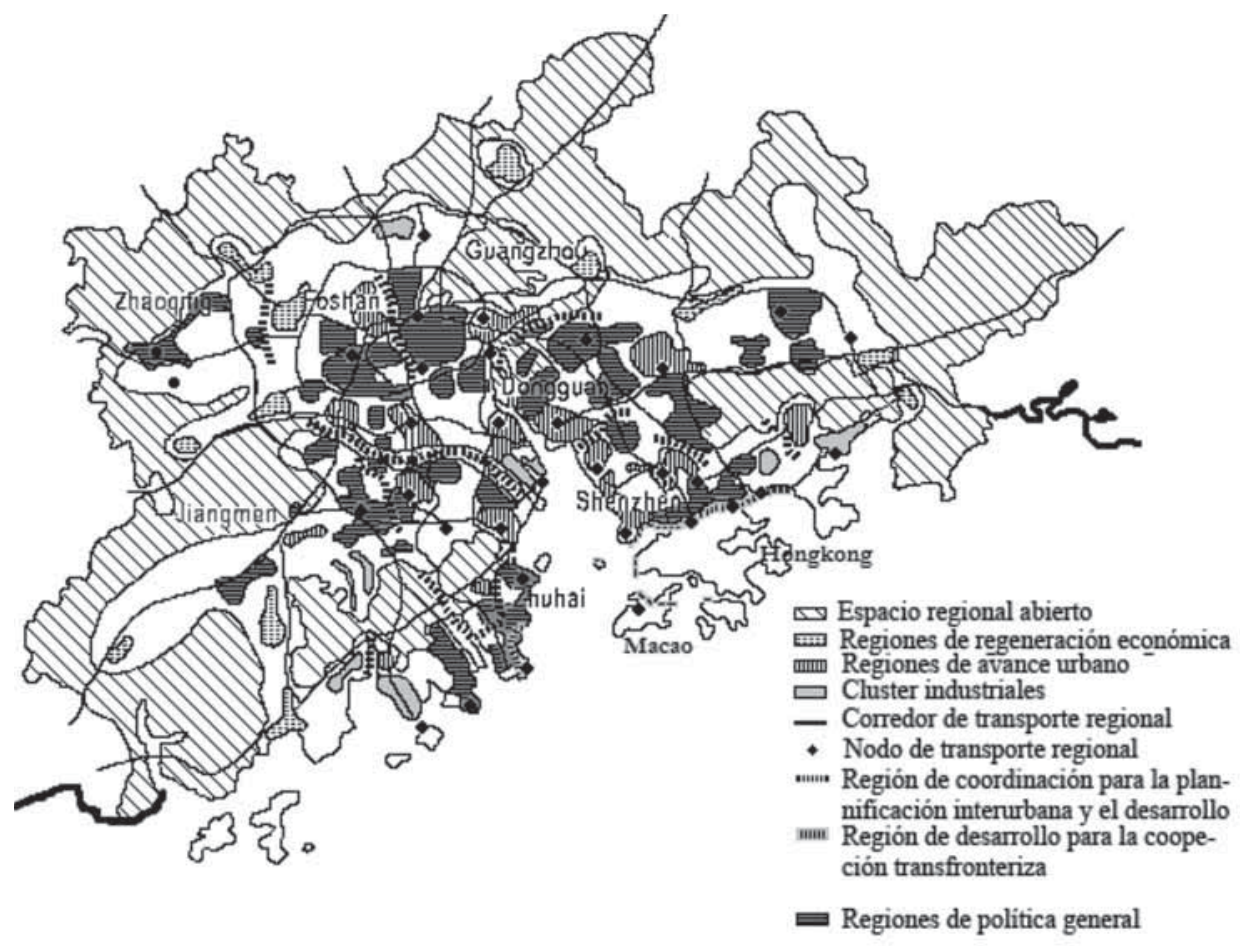

Fuente: Xu, J. y People's Government of Guangdong Province, 2005.

Estudios Geográficos, Vol. LXXIII, 272, pp. 273-307, enero-junio 2012

ISSN: 0014-1496, eISSN: 1988-8546, doi: 10.3989/estgeogr.201210 
encontrar un equilibrio entre el mercado y la gestión de gobierno, la equidad y la eficiencia del desarrollo regional (Feng, Wu y Zhu, 2008).

El Undécimo Plan Quinquenal (2006-2010) ha supuesto el último avance, dividiendo el espacio de todo el territorio nacional en cuatro tipos de regiones funcionales: el área de desarrollo óptimo, el área clave de desarrollo, el área de desarrollo limitado y el área de desarrollo prohibido. Los principales objetivos de esta división son aliviar el problema de la gradualmente agravada limitación de los recursos y el medio ambiente; la separación adecuada de la división administrativa, mejorando la gestión espacial del territorio nacional y planificar la región en su conjunto para mejorar la eficiencia del desarrollo. Este es un paso más de China para estandarizar las órdenes en el desarrollo espacial y formar estructuras racionales para la regionalización (Fourth Conference of The Tenth National People's Congress, 2006).

Sigue el principio de que una región tiene principalmente tres tipos de funciones: económica, social y ecológica. Estos grandes ámbitos funcionales fomentan sus propias fortalezas y limitan las debilidades, eligiendo los enfoques de desarrollo más adecuados a sus necesidades. Esto evita la sobreexplotación de los recursos y el daño al medio ambiente, y desempeña un papel vital en la consecución de equilibrio espacial para el desarrollo regional sostenible (Ma y $\mathrm{Li}, 2009$ ). Con este plan se plantea que la zonificación por funcionalidades pone el énfasis en la orientación del doble objetivo de la igualdad y de la eficiencia que anteriormente habían sido tratados de manera excluyente. Por un lado, es beneficioso para mejorar la competitividad internacional de China, fortaleciendo la capacidad de desarrollo de las zonas de aglomeración urbana a través de la optimización y el crecimiento. Por otro lado, mejorando la calidad de vida de las personas en las zonas donde el desarrollo esté prohibido o restringido, por medio de transferencias financieras, es beneficioso para la reducción de disparidades regionales en los servicios públicos básicos y la consecución de la igualdad en términos de desarrollo (Fan y Li, 2009).

\section{Crecimiento eConómico y Desarrollo Regional}

El ascenso de China como potencia económica mundial comenzó con el periodo de la «Reforma y Apertura» en la zona costera (Cabrillac, 2003; Gipouloux, 2005). Desde entonces el país ha confiado principalmente en las exportaciones emitidas desde este espacio para garantizar el crecimiento, basado principalmente en su casi ilimitado aporte de mano de obra barata. Con su acceso a la Organización Mundial de Comercio en 2001, una cuarta parte del crecimiento de China se generó por las exportaciones y las inversiones ligadas 
a ellas. China se convirtió no solo en el eje de la división del trabajo en Asia, sino en una fábrica mundial. Y su crecimiento está trayendo cambios en la economía mundial y en las relaciones internacionales (Fabre, 2009; Gaulier, Lemoine y Ünal-Kesenci, 2006, 2007 y 2011).

Pero el fuerte crecimiento económico de China se asoció con una aceleración de su comercio exterior y con un crecimiento de los desequilibrios internos. La crisis asiática de los años noventa y la crisis mundial que explotó a finales de 2008 han revelado la vulnerabilidad del modelo de crecimiento del país. La economía china mantiene una fuerte dependencia de los mercados exteriores y tiene que cambiar progresivamente hacia un modelo de crecimiento centrado en la demanda interna. Sin embargo este proceso llevará tiempo (Gaulier, Lemoine y Ünal-Kesenci, 2011).

El mayor impacto de la crisis ha sido en las pequeñas y medianas empresas con mano de obra intensiva orientadas a la exportación, situadas en la zona costera y vulnerables a los cambios del mercado (Gaulier, Lemoine y Ünal-Kesenci, 2007). Los flujos de trabajadores emigrantes procedentes de las provincias del interior se están revertiendo. La situación, sin embargo, es menos dramática para los sectores de alto valor añadido. Las autoridades nacionales están usando abiertamente esta oportunidad para reestructurar la economía, esta vez en favor de los sectores intensivos en tecnología y de mayor valor añadido, y para organizar el retorno de emigrantes a sus provincias de origen con el fin de fomentar el desarrollo de las regiones más pobres (Fabre, 2009).

La cuestión relevante es que China ha elegido la política rural como el principal nivelador de la demanda interna: el consumo rural representa cerca de un tercio del total, a pesar de que la población que aún vive en el campo es el $60 \%$. En este sentido, en 2008, China adoptó una nueva reforma extendiendo los contratos de arrendamiento de las tierras a los agricultores desde el tipo de 30 años a uno abierto y protegiendo los derechos de uso de las tierras, incluyendo el derecho de transferencia. Ante la situación de años recientes, en la que gran cantidad de tierras cultivables habían sido abandonadas o sub-explotadas - unos 130 millones de personas emigraron a las ciudades para trabajar en las fábricas o en el sector de la construcción-, la nueva política puede permitir a los agricultores usar sus tierras como aval para acceder a crédito o alquilarlas cuando migran. La adopción de esta nueva política puede incrementar los ingresos rurales, disminuir la brecha entre el sector urbano y rural, estimular la urbanización sobre una base sólida e incrementar la productividad y contribuir a disminuir la polarización social actual, en la que el $10 \%$ de las familias más ricas poseen más del $40 \%$ de la riqueza, y el $10 \%$ de los más pobres solo el 2\% (Fabre, 2009). 


\section{CONCLUSIONES}

En primer lugar se quiere precisar que el desarrollo regional en China tiene como objeto de preocupación otros temas como la política militar y de defensa (Brisset, 2002; Cabestan, 2009), en la que está incluida la preocupación sobre la reunificación de Taiwan (Cabestan y Vermander, 2005; Cabestan, 2007). Sin embargo estos aspectos no han sido tratados en este artículo.

Un primer análisis de cómo ha sido la toma en consideración de la cuestión regional en la República Popular China nos muestra que el principal error cometido es la dinámica de continua corrección del sistema: cuando una innovación estaba en curso han surgido nuevos problemas y volvía a recuperarse la vieja estructura o se volvía a modificar. Así que la cuestión más importante a la que hay que prestar atención en el futuro proceso de desarrollo regional son las condiciones específicas de cada región cuando se inicia una reforma, evitando proyectos generalistas e incluso el establecimiento de la misma norma para toda la nación, ya que China en sí misma es una realidad altamente compleja. Por ejemplo, la reforma actual del sistema Province Leads County, es beneficiosa para algunas regiones a fin de que puedan disminuir los niveles administrativos, y consecuentemente reducir el gasto administrativo. Sin embargo, en algunas regiones con gran territorio y población no puede ser aplicado, ya que es muy difícil para una provincia gobernar demasiados condados. La solución en este caso podría pasar por mantener su sistema City Leads County o bien dividir la provincia en mas órganos provinciales.

Como hemos visto, desde su fundación, la República Popular China ha invertido grandes esfuerzos buscando la formula más adecuada para lograr un mejor desarrollo regional. Pero China es un país de extensos territorios y altamente poblado en el que aún persisten muchos problemas sin resolver. En el ámbito administrativo, los aspectos que necesitan mejoras incluyen: la escala de gestión administrativa, su autoridad y su función, su modelo institucional y la dotación de personal, establecer su nivel y la relación de subordinación administrativa. Por ejemplo, la dimensión de las regiones autónomas de Mongolia Interior es estrecha y larga, se distribuye en más de 2.400 kilómetros de oeste a este. Desde el punto de vista del desarrollo a largo plazo, es necesaria la fragmentación y la nueva combinación de las provincias para reducir la extensión territorial con el fin de mejorar la eficiencia de la administración local. Todo lo anterior significa que se necesita una gran cantidad de trabajo en el futuro para completar la construcción del sistema de división administrativa. Sin embargo, como apunta J. Z. H. Fan (2007), la innovación y el ajuste de la división administrativa es una cuestión complicada y sensible en la que se de- 
ben tener en cuenta múltiples factores como el desarrollo económico, las condiciones geográficas, la densidad de población, el mantenimiento de la estabilidad social y la armonía étnica, entre otras cuestiones.

En el campo de las políticas de planificación regional, el Undécimo Plan Quinquenal ha fijado el patrón de desarrollo de un futuro próximo, los dos puntos clave en que se basa son: romper adecuadamente la limitación administrativa y promover el movimiento libre de factores de producción entre las regiones; el desarrollo de más aglomeraciones urbanas para irradiar y estimular el desarrollo económico en el área rural. Es muy importante terminar el mapa de la regionalización de los cuatro tipos de regiones funcionales para ayudar al logro de estos objetivos. En resumen, el trabajo desde una perspectiva de desarrollo regional, como ha sido realizado en el último período, sería más adecuada y útil para encontrar una regionalización que ayude al desarrollo integral de todos los territorios.

Recibido: $26 / 11 / 2010$

Aceptado: 21/06/2011

\section{BIBLIOGRAFÍA}

Ba, T. (1993): Survive, sustenance, development - Mao's view of human rights. Beijing, China Politics and Law University Press.

Bao, SH. M.; Chang, G. H., Sachs J. D. y Woo W. T. (2002): "Geographic factors and China's regional developsment under market reforms 1978-1998". China Economic Review, 13, pp. 89-111.

Brisset, J. V. (2002): China, an encircled power? Paris, Institut de Relations Internationales et Strategiques.

Bu, Q. H. (2009): Historical evolution of China's administration division, Map. 2. Beijing, China Map Press.

Cabestan, J. P. (2007): "A paper prepared for the international conference - 'One Country, Two Systems, Three Legal orders'- Perspectives and Evolution”. ERENLAI Magazine, February, 12.

Cabestan, J. P. (2009): "China's foreign and security policy decision making processes under Hu Jintao". Journal of Current Chinese Affairs, 38/3, pp. 63-97.

Cabestan, J. P. y Vermander, B. (2005): La Chine en quete de ses frontieres: La confrontation Chine-Taiwan. Paris, Presses de Sciences.

Cabrillac, B. (2003): Economie de la China. Paris, Presses Universitaires de France.

Chang, S. E. (1997): "Regional economic structure and capital investment in the built physical environments". Papers in Regional Science, 76/4, pp. 385-467. 
Chen, CH. (1999): "Reviews on the economic opening of the borderland around China's west". Journal of Xiangtan Normal University (Social science ed), 6.

Chen, SH. Y. y Zhang B. X. (2009): "Changes and prospects of China administrative division and relationships between levels of government over the past 60 years". Journal of Zhejiang Technology and Business University, 5.

China Map Press (1995): Situation of the Liberation of Mainland. Beijing, China Map Press.

China Map Press (2009): Evolution of Chinese administrative division. Map, 2. Beijing, China Map Press.

China Map Press (2009, September 16): On geographical regions of China. Beijing, China Map Press. Disponible en: http://www.chinamap.com/HTML/baodaoshuoming2.htm (Fecha de consulta: 25/01/2012).

Chun, Y. (2006): "The Pearl River Delta and Hong Kong: an evolving cross-boundary region under One Country, Two Systems". Habitat International, 30/2, pp. 61-86.

Constitution of People's Republic of China (1954). China, National People's Congress. Constitution of People's Republic of China (1975). China, National People's Congress. Constitution of People's Republic of China (1978). China, National People's Congress. Constitution of People's Republic of China (1982). China, National People's Congress. CPC Central Committee - State Council (1983a): Instructions about questions on reform of party and government organs at local level. China.

CPC Central Committee - State Council (1983b): Instructions about repeal people's commune and reinstruct People s Government at village level. China.

CPC Central Committee - State Council (1999): Announcement of the Fifteenth CPC Forth Plenary Session. Disponible en: http://news.cqnews.net/cqnews_chat/200909/ t20090915_3595108.htm (Fecha de consulta: 25/01/2012).

CPC Central Committee - State Council (1999): Announcement of the Fifteenth CPC Forth Plenary Session. China.

CPC News (1949, March 5): "Second Plenary Session of the Seventh Central Committee". Disponible en: http://dangshi.people.com.cn/GB/165617/index.html (Fecha de consulta: 25/01/2012).

Dai, J. L. (1999): "Review and summary of administrative division in 50 years". China Territorial (Administration Section), 5.

Dai, J. L. (2000): Chinese system of city. Beijing, China Map Press.

Department of Civil Administration of the People's Republic of China (1986-2006): Manuals of Administrative Division of the People's Republic of China. Beijing, China Map Press.

Department of Civil Administration of the People's Republic of China (1998): History of Administrative Division of the People's Republic of China 1949-1997. China, Social Press.

Dong, J. y Liu, W. G. (2007): "The beginning and end of the system of major administrative region in the initial of People's Republic of China". Vicissitudes, 2.

Editorial Committee on Party Literature of the Central Committee (1993): Selected works of Deng XioaoPing, 3. Beijing, People's Press. 
Fabre, G. (2002): China, crisis y cambio. París, LHarmattan.

Fabre, G. (2009): The twilight of "Chimerica"?: China and the collapse of the American model. Working Paper, Department of economics Ca Foscari University of Venice.

Fan, C. C. (1995): "Of belts and ladders: State policy and uneven regional development in Post-Mao China". Annuals of the Association of American Geographers, 85, pp. 421-449.

Fan, C. C. (1997): Uneven development and beyond: regional development theory in postMao China. Malden, Blackwell Publishers.

Fan, C. C. (2004): Book review of Developing China's West: A Critical Path to Balanced National Development. Hong Kong, The Chinese University Press of Hong Kong.

Fan, J. y Li, P. X. (2009): "The scientific foundation of Major Function Oriented Zoning in China". Journal o Geographical Sciences, 19, pp. 515-531.

Fan, J. ZH. (2007): "The state and problem of administrative division of China". Study of Contemporary China, 1.

Feng, CH. CH.; Wu, G. y Zhu J. J. (2008, June 20): "Regional planning in China" en: Megaregions and Trasportation. Planning Framework Symposium. Atlanta, Georgia Tech.

Fourteenth CPC Fifth Plenary Session (1996): Suggestions about the Ninth Five-year Plan of national economic and social development and long-range objectives by the year 2010. Disponible en: http://www.allzg.com/n3210c189.aspx (Fecha de consulta: 25/01/2012).

Fourth Conference of the Sixth National People's Congress (1986): The People's Republic of China's Eleventh Five-year Plan of National Economic and Social Development. Beijing, Fourteenth CPC Fifth Plenary Session.

Galán, I. (1977): Mao y la revolución China. Madrid, Editorial Cuadernos para el Dialogo, Colección los Suplementos, 79, pp. 33-38.

Gaulier, G..; Lemoine, F. y Ünal-Kesenci, D. (2007): "China s integration in East Asia: Production sharing, FDI and high-tech trade". CEPII Working Paper 2007-09. Disponible en: www.cepii.fr (Fecha de consulta: 25/01/2012).

Gaulier, G..; Lemoine, F. y Ünal-Kesenci, D. (2011): China's foreign trade in the perspective of a more balanced economic growth. CEPII Working Paper 2011-03. www.cepii.fr (Fecha de consulta: 25/01/2012).

Gaulier, G.; Lemoine, F. y Ünal-Kesenci, D. (2006): "China's Emergence and the Reorganisation of Trade Flows in Asia". CEPII Working Paper 2006-05. Disponible en: www.cepii.fr (Fecha de consulta: 25/01/2012).

Gernet, J. (2006): A history of Chinese civilization (2 volume set). London, The Folio Society.

Gernet, J. (2008): El mundo chino. Barcelona, Editorial Crítica.

Gipouloux, F. (2005): La Chine du 21e siecle: Une nouvelle superpuissance?. Paris, Armand Colin.

Government Administration Council (1951): Instruction about construction of people's democratic regime. Beijing, Government Administration Council. 
Guo, J. Y. (Producer) (2007): Renewal road [Television broadcast]. Beijing, China International TV Corporation.

Han, S. S. y Wong, S. T. (1994): "The influence of Chinese reform and pre-reform policies on urban growth in the 1980s". Urban Geography, 15/6, pp. 537-564.

History Department of People's Education Publishing House and Education Map Centre of China Map Press (2003): Historical atlas of modern China, 1. Beijing, China Map Press.

Hu, X. J. (2006): Historical evolution of China in unified territory. Map, 6. Beijing, China Map Press.

Hua, W. (2003): "On reconstruction of state and prefectures". Select Writings of Government Officials, 10.

Li, J. L. y Deng, CH. SH. (2009): "The development of Chinese administrative division and prospect for the future in new China". Economic Geography, 12.

Lin, J. B. (1995): "The evolution of China's regional development policies". Regional Development Studies, 1, pp. 55-82.

Liu, CH. Y. (2006): "On the administrative divisions of Chinese township's historical evolution and development". Scientific Socialism, 4.

Lu, D. D. (1995): Regional development and its spatial structure. Beijing, Science Press.

Ma, H. X. y Li, H. L. (2009, June): "Discussions about some problems to the western region of the main functions division and construction, taking Xinjiang Province as the example". Areal Research and Development, 28/3.

Ministry of Construction City Planning Division (1990): Explanation about Law of Urban Planning. Beijing, Public Publishing House.

Ministry of Finance (2009): Proposal about promote reform of province governs county's finance system. Beijing, Ministry of Finance.

People s Government of Guangdong Province (2005): Development plan of Pearl River Delta urban agglomeration 2004-2020. Guangdong, People's Government of Guangdong Province.

Qu, G. Y. y Chai, H. Y. (2009, February): "Analysis and research about Chinese administrative division changes in recent 20 years". Yinshan Academic Journal, 22/1.

Resolutions on People's Communes (1958): Bulletin of Sixth Plenary Session of the Eighth Central Committee. Beijing, Sixth Plenary Session of the Eighth Central Committee.

Roberts B. H. y Murray A. T. (2002): "National and regional corporate spatial structure". The Annals of Regional Science, 36, pp. 347-368.

Roux, A. (2006): La Chine au XXe siecle. Paris, SEDES.

State Council (1986): Instructions about adjust standards of city establishment and conditions of city leads country. Beijing, State Council.

Sun, SH. H. y Yan, ZH. X. (1999): "China's Coastal Cities: development, planning and challenges". Pergamon, Habit At Intl., 23/2, pp. 217-229.

Williams, S. (Producer and Director) (1994): China trilogy: The Mao years, 1949-1976 [Documentary]. EEUU, AMBRICA Productions. 
Williams, S. (Producer and Director) y Dietz K. P. (Producer) (1989): China trilogy: China in revolution, 1911-1949 [Documentary]. EEUU, AMBRICA Productions.

World Bank (1997): China 2020: disparities in China. Washington DC, The World Bank.

$\mathrm{Xu}$, J. (N.D.): Inter-jurisdictional Cooperation and mega-city region governance in the

Pearl River Delta. Disponible en: http://www.grm.cuhk.edu.hk/eng/research/feature/interjurisPRD.html (Fecha de consulta: 25/01/2012). Hong Kong, Geography and Resource Management Faculty. Chinese University of Hong Kong.

Yagüe, J. L. y Díaz-Puente, J. M. (2008): "Tres siglos de planificación regional en Uruguay: lecciones de experiencia para afrontar los retos de desarrollo en el siglo xxI". Estudios Geográficos, LXIX/264, pp. 247-280.

Yeung, Y. M. y Hu, X. W. (eds.) (1992): China's Coastal Cities. Honolulu, University of Hawaii Press.

Yu, M. CH. (1998): "Over Chinese province system". Strategy and Management, 4.

Zhang, CH. G. (1999): On county field. Beijing, China Federation of literary and Culture publishing House.

Zhang, X. J. (1994): "The Reduce Province Campaign in Republic of China". The 21th Century, 10, p. 48.

Zheng, L. (2009): "How much authority has the 'Bigger City'-Investigation on the Constitution text-". National School of Administration Journal, 1.

\section{RESUMEN}

Desde la fundación de la República Popular China el Gobierno Central ha estado buscando la vía más adecuada para conseguir el desarrollo regional. En el período de Mao el enfoque adoptó una perspectiva principalmente administrativa, tratando de mejorar la estructura de división y de gobierno del país. Con el inicio del período de reforma y apertura, el acento cambia al diseño de estrategias de desarrollo regional, aunque con tendencias cambiantes que van desde las políticas redistributivas hasta el modelo de desarrollo asimétrico, y los esfuerzos por combatir las desigualdades entre regiones y entre áreas urbanas y rurales de China.

Este artículo realiza una revisión de los cambios producidos en China a través de todos estos procesos de regionalización, proporcionando un marco de de referencia para comprender las diferentes etapas y ayudar a estudios más específicos sobre este país.

Palabras Clave: desarrollo regional; República Popular China; división administrativa; planificación regional; regionalización.

\section{Abstract}

Since the foundation of the People's Republic of China, Chinese Central People's Government has always been seeking a more adequate way to achieve a better regional development. The emphasis of the Mao era was mainly focused on administrative perspective, intending to improve the scheme of dividing and governing the country. Since the Reform and Open-up, the emphasis shifted to refining the regional develop- 
ment strategies, the trends of which have changed from Maoist redistributive policies to Deng's uneven development model and to the efforts to battle the widening gap among regions and between urban and rural areas of China since Seventh Five-year Plan (1986-1990) until now. This article realizes a review of the undergoing process of China's regionalization, in order to provide a framework of reference for further studies about this field.

KEY WORDS: regional development; People's Republic of China; administrative division; regional planning; regionalization.

\section{RÉSUMÉ}

À partir de la fondation de la République populaire de Chine le gouvernement central a cherché le moyen le plus approprié pour atteindre le développement régional. Dans la période de Mao l'approche essentiellement adoptée fut un point de vue administratif, en essayant d'améliorer la structure de la division et le gouvernement du pays. Avec le début de la réforme et la période d'ouverture, les directives changent vers la conception de stratégies de développement régional, mais avec une évolution des tendances allant depuis les politiques de redistribution jusqu'au modèle de développement asymétrique, et les efforts pour lutter contre les inégalités entre les régions et entre les zones urbaines et rurales de La Chine.

Cet article passe en revue les changements en Chine à travers tous ces processus de régionalisation, en fournissant un cadre pour comprendre les différentes étapes et pour aider les études plus spécifiques sur ce pays.

Mots CLÉs: développement régional; République Populaire de Chine; division administrative; planification régionale; régionalisation. 